\title{
Variational Multiscale error estimators for the adaptive mesh refinement of compressible flow simulations
}

\author{
Camilo Bayona-Roa ${ }^{\mathrm{a}, \mathrm{b}}$, Ramon Codina ${ }^{\mathrm{a}, \mathrm{b},}$, Joan Baiges $^{\mathrm{b}, *}$ \\ ${ }^{a}$ Centre Internacional de Mètodes Numèrics en Enginyeria (CIMNE), \\ Edifici C1, Campus Nord UPC C/Gran Capità S/N 08034 Barcelona, Spain \\ ${ }^{b}$ Universitat Politècnica de Catalunya, Jordi Girona 1-3, \\ Edifici C1, 08034 Barcelona, Spain
}

\begin{abstract}
This article investigates an explicit a-posteriori error estimator for the finite element approximation of the compressible Navier-Stokes equations. The proposed methodology employes the Variational Multi-Scale framework, and specifically, the idea is to use the variational subscales to estimate the error. These subscales are defined to be orthogonal to the finite element space, dynamic and non-linear, and both the subscales in the interior of the element and on the element boundaries are considered. Another particularity of the model is that we define some norms that lead to a dimensionally consistent measure of the compressible flow solution error inside each element; a scaled $L^{2}$-norm, and the calculation of a physical entropy measure, are both studied in this work. The estimation of the error is used to drive the adaptive mesh refinement of several compressible flow simulations. Numerical results demonstrate good accuracy of the local error estimate and the ability to drive the adaptative mesh refinement to minimize the error through the computational domain.
\end{abstract}

Keywords:

Compressible Navier-Stokes equations, Variational Multi-Scale (VMS) method, Orthogonal Sub-Grid Scales (OSGS), A-posteriori local error estimation, Adaptive Mesh Refinement (AMR)

\section{Introduction}

The compressible Navier-Stokes equations, namely the conservation of mass, momentum, and energy, together with constitutive and thermodynamical relations, constitute a physical model that describes the compressible fluid flow phenomena. This model is able to represent the wide range of spatial and temporal flow scales typically encountered in engineering cases of interest. When numerically approximating these equations, the smallest flow scales (in turbulence or aeroacoustics, for example) must be modeled

\footnotetext{
* Corresponding author

Email addresses: cbayona@cimne.upc.edu (Camilo Bayona-Roa), ramon.codina@upc.edu (Ramon Codina), joan.baiges@upc.edu (Joan Baiges)

Preprint submitted to Computer Methods in Applied Mechanics and Engineering December 26, 2017
} 
with a high level of accuracy by numerical means, but one major source of error is the discretization error: the solution obtained with coarse meshes is often too inaccurate, and calculating over fine meshes is impractical considering the amount of computational effort. Adaptive Mesh Refinement (AMR) methods deal with this issue by dynamically re-configuring the initial mesh and changing its structure employing some type of criteria. The AMR involves two main steps: first, the decision of which elements to modify (mainly the ones contributing the most to the global solution error), and then, the adaptation of those selected elements. The focus of the present work is to investigate a local estimate of the approximation error that can be suitable for driving the AMR of compressible flow simulations. To this end, we exploit the Variational Multi-Scale (VMS) framework introduced in [1], that is typically used to stabilize the fluid flow equations (see for instance the review of the VMS applied to fluid problems in [2]).

The VMS method decomposes the solution space into a resolved component, that is captured by the finite discretization, and a sub-grid part, which is the remainder that cannot be represented by the finite grid. The original discrete problem is therefore equivalent to two sub-problems: one for each scale. Although the variational sub-grid scales (or subscales) have been also adopted for turbulence modeling (see for example $[3-5]$ and references therein), essentially, the role of the subscales is related to the error of the finite approximation. Indeed, the variational subscales vanish consistently as the discrete solution tends to the exact solution, so that, they have been identified with residual-based error estimators (e.g in [6]). The recent literature on variational subscales error estimators is too vast to survey here, but we mention the early works of variational subscales as a-posteriori error estimates in $[7,8]$, where a patch of the elements in the mesh was used to calculate the subscales by decoupling a global residual equation with a localization function, and the element-residual methods in $[9,10]$, which were able to provide implicit estimations with the subscales inside each element of the mesh (and in some cases on the element boundaries). The application of the subscales as explicit aposteriori estimators has been developed in $[6,11,12]$ from the subscales at the interior and at the boundaries of the element using element Green's functions. In the case of incompressible flow simulations, the subscales have been used as error estimators for AMR in [13] by using a non-dimensional norm of the velocity subscales inside the element. However, the explicit a-posteriori error estimation given by the variational subscales has been less accurate than implicit goal-oriented methods for compressible flow problems in [14-18], or than energy norm estimates using implicit residual methods in [19, 20], but cheaper, since the solution of additional differential equations is not required. We refer to the review article by [21] for a deeper understanding of the variational subscales as explicit a-posteriori error estimators.

The present approach is similar to the one in [12], where an explicit a-posteriori estimation of the error for the Euler and Compressible Navier-Stokes equations was constructed. We also derive an explicit a-posteriori error estimator from the VMS framework, but our approach offers three different and novel ingredients. First, we define the space where the variational subscales live as the space orthogonal to the finite element space, contrary to the most common choice to define it equal to the space of the finite element residuals. This is the so-called Orthogonal Sub-Grid Scales (OSGS) method, first introduced in [22], and which has been recently applied as an explicit a-posteriori error estimator for the elastic problem of solids in [23]. We also include the temporal tracking of the subscales, to what is referred in [24] as the dynamic subscales, and account for 
including the subscales into all of the non-linear terms of the problem. In consequence, we refer to the subscales as to be orthogonal, dynamic and non-linear. Second, we model the effect of the subscales inside each element from the perspective of a Fourier analysis, as developed in [25, 26], instead of using Green's functions in [1, 6, 11, 12]. Third, we model the subscales at the boundaries of the elements, as first presented in [27], and calculate them as part of the error estimator.

In this article, the error estimation given by the variational multiscale is intended to drive the adaptive mesh refinement process of compressible flow simulations. The error estimate must be well constructed in a dimensional sense, so that, the contribution of the subscales of the different equations (i.e. the subscales of the mass, momentum and energy equations) into the estimation must be consistent. Several norms can be used in this regard. The $L^{1}$ and $L^{2}$ norms of the variational subscales of velocity in [13] and of the subscales of the separated compressible equations in [12], and the energy norm of the variational compressible problem in [20], have been previously applied to estimate the error inside each element. Since the error estimation given by each separated variational subscales (of the compressible equations) can vary greatly, and therefore, each refined mesh driven by the estimation of a single subscale may lead to unbalanced errors between the multiple variables of the problem, we propose to calculate the error estimation accounting for all the variational subscales. To this end, we propose two different approaches that provide dimensionally consistent measures of the solution error inside each element. The first approach is to calculate a scaled $L^{2}$-norm of the variational subscales inside the element, and on the element boundaries, as well. This approach goes in line to the one presented in [23]. The second approach is to compute the relative $L^{2}$ error norm of the physical entropy (for ideal gases) calculated using the variational subscales inside the element. The error estimation given by these two norms is used by the adaptive algorithm to drive the addition or removal of elements where the error is outside a given threshold. The $h$-adaptive method adopted here, including the refinement strategy and the data structures needed, has been presented in [28] and implemented in the RefficientLib software. Nevertheless, the proposed strategy is applicable to $p$, $r$, and $h p$ adaptive mesh refinement techniques, although these are not explored in the present article. The AMR simulations demonstrate the ability of the variational subscales to lead an accurate approximation, provided the error estimation is kept always below the threshold inside the computational domain.

The paper is divided into the following parts. In Section 2, the compressible flow problem is presented. Next, the variational multi-scale finite element formulation of the problem is described in Section 3. The design of the variational subscales error estimator is presented in Section 4. Some numerical examples, including subsonic and supersonic problems in two and three dimensions, are demonstrated in Section 5. Finally, in Section 6 some conclusions close the article.

\section{Problem definition}

In this section, we present the governing equations for the compressible fluid flow problem, i.e., the compressible Navier-Stokes equations. For doing so, the strong form of the problem is described in the first part of the section. Then, we transform the strong form of the problem into a quasi-linear form that allows us to deal with the non-linearities 
of the problem. The weak form of the compressible problem is introduced at the last part of the section.

\subsection{Compressible Navier-Stokes equations in strong form}

Consider a spatial domain $\Omega \subset \mathbb{R}^{d}$, being $d$ the number of space dimensions ( $d=2$ or 3 ), and the time interval $\left(0, t_{f}\right)$. Let $t \in\left(0, t_{f}\right)$ be a given time instant in the temporal domain, and $\boldsymbol{x} \in \Omega$ a given point in the spatial domain. Let $\Gamma$ be the boundary of the domain $\Omega$, and $\boldsymbol{n}$ the geometric unit outward normal vector on $\Gamma$. We split $\Gamma$ into two sets: the Dirichlet boundary denoted as $\Gamma_{G}$, and the Neumann boundary denoted as $\Gamma_{N}$. The strong form of the initial and boundary-value problem consists of finding the solution vector $\boldsymbol{U}: \Omega \times\left(0, t_{f}\right) \rightarrow \mathbb{R}^{d+2}$, where $d+2$ is the number of unknowns (the same as equations of the system), such that for the given Dirichlet boundary conditions $\boldsymbol{U}_{G}: \Gamma_{G} \times\left(0, t_{f}\right) \rightarrow \mathbb{R}^{d+2}$ and the Neumann boundary conditions $\boldsymbol{H}: \Gamma_{N} \times\left(0, t_{f}\right) \rightarrow \mathbb{R}^{d+2}$, the following compressible Navier-Stokes equations are satisfied:

$$
\begin{aligned}
\partial_{t} \boldsymbol{U}+\partial_{j} \boldsymbol{E}_{j}(\boldsymbol{U})+\partial_{j} \boldsymbol{G}_{j}(\boldsymbol{U}) & =\boldsymbol{F} & & \text { in } \Omega \subset \mathbb{R}^{d}, t \in\left(0, t_{f}\right), \\
\boldsymbol{D}(\boldsymbol{U}) & =\boldsymbol{D}\left(\boldsymbol{U}_{G}\right) & & \text { on } \Gamma_{G}, \quad t \in\left(0, t_{f}\right), \\
\boldsymbol{B}(\boldsymbol{U}) & =\boldsymbol{H} & & \text { on } \Gamma_{N}, \quad t \in\left(0, t_{f}\right), \\
\boldsymbol{U} & =\boldsymbol{U}^{0}(\boldsymbol{x}) & & \text { in } \Omega \subset \mathbb{R}^{d}, \quad t=0 .
\end{aligned}
$$

The Eulerian time derivative and $\partial / \partial x_{j}$ are indicated in short notations $\partial_{t}$ and $\partial_{j}$, respectively, and the usual summation convention is used over repeated indices. The Dirichlet boundary operator $\boldsymbol{D}(\cdot)$ is used to impose the components of $\boldsymbol{U}$ on different parts of $\Gamma$. The Neumann boundary conditions are given by the operator $\boldsymbol{B}(\cdot)$. Note that with our notation $\Gamma_{G}$ and $\Gamma_{N}$ may overlap.

The vector $\boldsymbol{U}=\left(\rho, \boldsymbol{m}, e_{\mathrm{tot}}\right)^{\top}$ denotes a vector of conservative variables, density $\rho$, momentum $\boldsymbol{m}=\rho \boldsymbol{u}$, and total energy $e_{\text {tot }}=\rho(e+\boldsymbol{u} \cdot \boldsymbol{u} / 2)$, where $\boldsymbol{u}$ stands for the velocity vector, and $e$ is the internal energy. The convective flux in the $j$ th-direction, $j=1, \ldots, d$, is defined as

$$
\boldsymbol{E}_{j}(\boldsymbol{U})=\left(\rho u_{j}, \rho u_{j} u_{1}+p \delta_{1 j}, \rho u_{j} u_{2}+p \delta_{2 j}, \rho u_{j} u_{3}+p \delta_{3 j}, u_{j}\left(e_{\text {tot }}+p\right)\right)^{\top},
$$

where $p$ is the pressure and $\boldsymbol{I}=\left[\delta_{i j}\right]$ is the identity or Kronecker tensor. The diffusive flux in the $j$ th-direction, $j=1, \ldots, d$, is

$$
\boldsymbol{G}_{j}(\boldsymbol{U})=\left(0,-\sigma_{j 1},-\sigma_{j 2},-\sigma_{j 3},-u_{i} \sigma_{i j}+q_{j}\right)^{\top},
$$

where $\boldsymbol{\sigma}$ is the viscous stress tensor, $\sigma_{i j}=\mu\left(\partial_{j} u_{i}+\partial_{i} u_{j}\right)-\frac{2}{3} \mu\left(\partial_{l} u_{l}\right) \delta_{i j}$, and $\boldsymbol{q}$ is the heat flux vector, $q_{i}=-\lambda \partial_{i} T$. Here $\mu$ is the viscosity, $\lambda$ is the thermal conductivity and $T$ is the temperature of the fluid. The vector of source terms is defined as

$$
\boldsymbol{F}(\boldsymbol{U})=(0, \rho \boldsymbol{f}, \rho \boldsymbol{f} \cdot \boldsymbol{u}+\rho r)^{\top},
$$

where $f$ is a body force vector, and $r$ is a heat source/sink term.

In the previous relations the caloric equation $e=c_{v}(T) T$ and the ideal law for gases $p=\rho R T$ are used to calculate the pressure and the acoustic speed $c=\sqrt{\gamma p / \rho}$, where the 
specific heat at constant volume $c_{v}(T)$ and the specific heat at constant pressure $c_{p}(T)$ are thermodynamic properties of the fluid, $\gamma=c_{p} / c_{v}$ is the ratio between the specific heats, and $R=c_{p}-c_{v}$ is the specific gas constant. The non-dimensional Mach number $M=|\boldsymbol{u}| / c$ is used to calculate the compressibility regime.

\subsection{Quasi-linear form of the problem}

Different formulations of the Navier-Stokes equations can be used, e.g. one can take $\boldsymbol{U}$ as the conservation variables, as done before, or one can also take $\boldsymbol{U}=(p, \boldsymbol{u}, T)^{\top}$, leading to the primitive variables formulation. Any of such formulations can be written in quasi-linear form as

$$
\boldsymbol{A}_{0}(\boldsymbol{U}) \partial_{t} \boldsymbol{U}+\mathcal{L}(\boldsymbol{U} ; \boldsymbol{U})=\boldsymbol{F} \quad \text { in } \quad \Omega \subset \mathbb{R}^{d}, t \in\left(0, t_{f}\right),
$$

together with appropriate boundary and initial conditions. Here we have introduced the non-linear operator $\mathcal{L}$, which is defined as

$$
\mathcal{L}(\widehat{\boldsymbol{U}} ; \boldsymbol{U})=\boldsymbol{A}_{j}(\widehat{\boldsymbol{U}}) \partial_{j} \boldsymbol{U}-\partial_{k}\left(\boldsymbol{K}_{k j}(\widehat{\boldsymbol{U}}) \partial_{j} \boldsymbol{U}\right) .
$$

Matrices $\boldsymbol{A}_{0}(\boldsymbol{U}), \boldsymbol{A}_{j}(\boldsymbol{U})$, and $\boldsymbol{K}_{k j}(\boldsymbol{U})$, for $k, j=1, . ., d$, are $(d+2) \times(d+2)$ matrices that depend upon $\boldsymbol{U}$, and that are appropriately defined for each type of formulation, as described in Appendix A. Both the conservative and the primitive formulations will be applied in the next sections of the article.

\subsection{Weak form of the problem}

Let $\mathcal{W}$ be the space of functions where, for each $t \in\left(0, t_{f}\right)$, the unknowns are well defined, with the appropriate regularity that we will not analyze here. Let us also denote by $(\cdot, \cdot)_{\omega}$ the integral of the product of two functions (scalar or vector-valued) in a domain $\omega$, omitting the subscript when $\omega=\Omega$. Introducing the form

$$
A(\boldsymbol{U} ; \boldsymbol{V}, \boldsymbol{W}):=\left(\boldsymbol{V}, \boldsymbol{A}_{j}(\boldsymbol{U}) \partial_{j} \boldsymbol{W}\right)+\left(\partial_{k} \boldsymbol{V}, \boldsymbol{K}_{k j}(\boldsymbol{U}) \partial_{j} \boldsymbol{W}\right),
$$

the variational form of the problem can be written as: find $\boldsymbol{U}:\left(0, t_{f}\right) \rightarrow \mathcal{W}$ such that

$$
\begin{aligned}
\left(\boldsymbol{V}, \boldsymbol{A}_{0}(\boldsymbol{U}) \partial_{t} \boldsymbol{U}\right)+A(\boldsymbol{U} ; \boldsymbol{V}, \boldsymbol{U}) & =(\boldsymbol{V}, \boldsymbol{F})+(\boldsymbol{V}, \boldsymbol{H})_{\Gamma_{N}}, & & t \in\left(0, t_{f}\right), \\
(\boldsymbol{V}, \boldsymbol{U}) & =\left(\boldsymbol{V}, \boldsymbol{U}^{0}\right), & & t=0,
\end{aligned}
$$

for all $\boldsymbol{V}$ in the adequate test functions space. The Neumann boundary operator is given by the diffusive fluxes

$$
\boldsymbol{B}(\boldsymbol{U})=-n_{k} \boldsymbol{K}_{k j}(\boldsymbol{U}) \partial_{j} \boldsymbol{U},
$$

although part of the convective term could also be integrated by parts and contribute to the Neumann boundary conditions, in particular, the pressure term. 


\section{Finite element formulation}

In this section, the finite element formulation of the compressible problem is described. The VMS formulation of the compressible problem that has been presented in [26, 29] is recalled in the section. As it will be explained, the VMS framework is used for stabilizing the finite element approximation and allows us to use arbitrary interpolation spaces for the different variables of the problem. The finite element equation is described first. Then, we focus the attention on the solution of the subscales; we address the equation for the subscales at the interior of the element, and later, the approximation for the subscales at the element boundaries is addressed. Finally, we describe the time integration scheme that is used for advancing in time at the end of the section.

\subsection{Variational Multi-Scale framework}

Let us first consider a finite-element partition $\mathcal{T}_{h}=\{K\}$ of the domain $\Omega$. The diameter of the element partition is denoted by $h$. We define the test functions space $\mathcal{W}_{h} \subset \mathcal{W}$ as made of continuous piecewise polynomial functions in space. The Galerkin approximation to problem (11)-(12) can be stated as follows: find $\boldsymbol{U}_{h}:\left(0, t_{f}\right) \rightarrow \mathcal{W}_{h}$ such that

$$
\begin{aligned}
\left(\boldsymbol{V}_{h}, \boldsymbol{A}_{0}\left(\boldsymbol{U}_{h}\right) \partial_{t} \boldsymbol{U}_{h}\right)+A\left(\boldsymbol{U}_{h} ; \boldsymbol{V}_{h}, \boldsymbol{U}_{h}\right) & =\left(\boldsymbol{V}_{h}, \boldsymbol{F}\right)+\left(\boldsymbol{V}_{h}, \boldsymbol{H}\right)_{\Gamma_{N}}, & t \in\left(0, t_{f}\right), \\
\left(\boldsymbol{V}_{h}, \boldsymbol{U}_{h}\right)=\left(\boldsymbol{V}_{h}, \boldsymbol{U}^{0}\right), & & t=0,
\end{aligned}
$$

for all $\boldsymbol{V}_{h} \in \mathcal{W}_{h}^{0}$, the discrete space of test functions (i.e., with components vanishing where Dirichlet conditions are prescribed on the boundary).

This approximation suffers from instability problems, which vary according to the way to construct $\mathcal{W}_{h}$ (e.g. in the case of equally interpolating spaces).

The VMS framework introduced in [1], has been established for overcoming this issue. The idea of the VMS framework is to decompose the space of the unknowns into a finitedimensional space $\mathcal{W}_{h}$, and an infinite-dimensional one, $\widetilde{\mathcal{W}}$, so that $\mathcal{W}=\mathcal{W}_{h} \oplus \widetilde{\mathcal{W}}$. The unknown and the test functions are accordingly split as $\boldsymbol{U}=\boldsymbol{U}_{h}+\widetilde{\boldsymbol{U}}$ and $\boldsymbol{V}=\boldsymbol{V}_{h}+\widetilde{\boldsymbol{V}}$, respectively. We shall refer to functions in $\mathcal{W}_{h}$ as the resolved scales and to functions in $\widetilde{\mathcal{W}}$ as the error or subscales.

Equation (11) can be equivalently written as the system of equations

$$
\left(\boldsymbol{V}_{h}, \boldsymbol{A}_{0}(\boldsymbol{U}) \partial_{t} \boldsymbol{U}\right)+A\left(\boldsymbol{U} ; \boldsymbol{V}_{h}, \boldsymbol{U}\right)=\left(\boldsymbol{V}_{h}, \boldsymbol{F}\right)+\left(\boldsymbol{V}_{h}, \boldsymbol{H}\right)_{\Gamma_{N}},
$$

for all $\boldsymbol{V}_{h} \in \mathcal{W}_{h}^{0}, t \in\left(0, t_{f}\right)$, and

$$
\left(\widetilde{\boldsymbol{V}}, \boldsymbol{A}_{0}(\boldsymbol{U}) \partial_{t} \boldsymbol{U}\right)+A(\boldsymbol{U} ; \tilde{\boldsymbol{V}}, \boldsymbol{U})=(\tilde{\boldsymbol{V}}, \boldsymbol{F})+(\widetilde{\boldsymbol{V}}, \boldsymbol{H})_{\Gamma_{N}},
$$

for all $\widetilde{\boldsymbol{V}} \in \widetilde{\mathcal{W}}^{0}, t \in\left(0, t_{f}\right)$, and likewise for the initial condition, Eq. (12). In Eq. (17), $\widetilde{\mathcal{W}}^{0}$ is the space of subscale test functions. 


\subsubsection{Finite element equation}

We first analyze the equation for the finite element scale (16). The time-dependent terms involving the temporal derivatives in the Left-Hand-Side (LHS) of Eq. (16) can be split as

$$
\left(\boldsymbol{V}_{h}, \boldsymbol{A}_{0}(\boldsymbol{U}) \partial_{t} \boldsymbol{U}\right)=\left(\boldsymbol{V}_{h}, \boldsymbol{A}_{0}(\boldsymbol{U}) \partial_{t} \boldsymbol{U}_{h}\right)+\left(\boldsymbol{V}_{h}, \boldsymbol{A}_{0}(\boldsymbol{U}) \partial_{t} \widetilde{\boldsymbol{U}}\right)
$$

Similarly, the second term in the LHS of Eq. (16) can be split as

$$
A\left(\boldsymbol{U} ; \boldsymbol{V}_{h}, \boldsymbol{U}\right)=A\left(\boldsymbol{U} ; \boldsymbol{V}_{h}, \boldsymbol{U}_{h}\right)+A\left(\boldsymbol{U} ; \boldsymbol{V}_{h}, \tilde{\boldsymbol{U}}\right)
$$

For convenience, the first terms in $A\left(\boldsymbol{U} ; \boldsymbol{V}_{h}, \widetilde{\boldsymbol{U}}\right)$ can be integrated by parts, that is,

$$
\begin{aligned}
\left(\boldsymbol{V}_{h}, \boldsymbol{A}_{j}(\boldsymbol{U}) \partial_{j} \tilde{\boldsymbol{U}}\right)= & -\sum_{K}\left(\partial_{j}\left(\boldsymbol{A}_{j}^{\top}(\boldsymbol{U}) \boldsymbol{V}_{h}\right), \tilde{\boldsymbol{U}}\right)_{K} \\
& +\sum_{K}\left(n_{j} \boldsymbol{A}_{j}^{\top}(\boldsymbol{U}) \boldsymbol{V}_{h}, \tilde{\boldsymbol{U}}\right)_{\partial K}, \\
\left(\partial_{k} \boldsymbol{V}_{h}, \boldsymbol{K}_{k j}(\boldsymbol{U}) \partial_{j} \tilde{\boldsymbol{U}}\right)= & -\sum_{K}\left(\partial_{j}\left(\boldsymbol{K}_{k j}^{\top}(\boldsymbol{U}) \partial_{k} \boldsymbol{V}_{h}\right), \tilde{\boldsymbol{U}}\right)_{K} \\
& +\sum_{K}\left(n_{j} \boldsymbol{K}_{k j}(\boldsymbol{U})^{\top} \partial_{k} \boldsymbol{V}_{h}, \tilde{\boldsymbol{U}}\right)_{\partial K} .
\end{aligned}
$$

Note that these terms in (19) involve inter-element jumps. For continuous solution finite element spaces, the convective term jump at the element boundaries in the Right-HandSide (RHS) of (20) is continuous because it is a function of the variables and, therefore, its sum across adjacent element boundaries is zero. On the contrary, the diffusive term at the element boundaries in the RHS of (21) contains derivatives of the variables and it is discontinuous even for continuous finite element spaces.

If we introduce the formal adjoint $\mathcal{L}^{*}(\boldsymbol{U} ; \cdot)$ of the operator $\mathcal{L}(\boldsymbol{U} ; \cdot)$, which is

$$
\mathcal{L}^{*}\left(\boldsymbol{U} ; \boldsymbol{V}_{h}\right)=-\partial_{j}\left(\boldsymbol{A}_{j}^{\top}(\boldsymbol{U}) \boldsymbol{V}_{h}\right)-\partial_{j}\left(\boldsymbol{K}_{k j}^{\top}(\boldsymbol{U}) \partial_{k} \boldsymbol{V}_{h}\right),
$$

the term related to the subscales in the finite-element equation (19) can be written as

$$
A\left(\boldsymbol{U} ; \boldsymbol{V}_{h}, \tilde{\boldsymbol{U}}\right)=\sum_{K}\left(\mathcal{L}^{*}\left(\boldsymbol{U} ; \boldsymbol{V}_{h}\right), \tilde{\boldsymbol{U}}\right)_{K}+\sum_{K}\left(n_{j} \boldsymbol{K}_{k j}^{\top}(\boldsymbol{U}) \partial_{k} \boldsymbol{V}_{h}, \tilde{\boldsymbol{U}}\right)_{\partial K}
$$

We divide the approximation of the variational subscales: either we define the subscales in the interior of the element, or we define them at the element boundaries. The first term at the RHS of (23) is the stabilization term that includes the subscales in the interior of the element $\widetilde{\boldsymbol{U}}$, whereas, the second term is the element boundary term that relates the subscales at the internal and boundary edges, that we call $\widetilde{\boldsymbol{U}}_{E}$. We introduce some approximations to calculate the variational subscales; the way we deal with those approximations will be explained in the following paragraphs. In this work we also make the following simplification: we only account for the interior subscales in the solution of the finite element problem, whereas, the subscales at the boundary are calculated as part of the error estimator. 


\subsubsection{Subscales in the interior of the element}

It is readily seen that, after integrating by parts the diffusive term of the LHS of (17), the equation for the subscales can be written as

$$
\left(\tilde{\boldsymbol{V}}, \boldsymbol{A}_{0}(\boldsymbol{U}) \partial_{t} \widetilde{\boldsymbol{U}}+\mathcal{L}(\boldsymbol{U} ; \tilde{\boldsymbol{U}})\right)=\left(\tilde{\boldsymbol{V}}, \boldsymbol{F}-\boldsymbol{A}_{0}(\boldsymbol{U}) \partial_{t} \boldsymbol{U}_{h}-\mathcal{L}\left(\boldsymbol{U} ; \boldsymbol{U}_{h}\right)\right)
$$

At this point we introduce the main approximation

$$
\mathcal{L}(\boldsymbol{U} ; \tilde{\boldsymbol{U}}) \approx \boldsymbol{\tau}^{-1}(\boldsymbol{U}) \widetilde{\boldsymbol{U}}
$$

so that the application of the non-linear operator to the subscales is modeled by a matrix of stabilization parameters $\boldsymbol{\tau}^{-1}$ that depends over the unknowns. If $\widetilde{\boldsymbol{P}}$ denotes the $L^{2}$ projection onto the space of subscales, the equation for the subscales in the interior of the element (24) can be formally written as

$$
\boldsymbol{A}_{0}(\boldsymbol{U}) \partial_{t} \widetilde{\boldsymbol{U}}+\boldsymbol{\tau}^{-1}(\boldsymbol{U}) \tilde{\boldsymbol{U}}=\widetilde{\boldsymbol{P}}\left[\boldsymbol{R}\left(\boldsymbol{U} ; \boldsymbol{U}_{h}\right)\right]
$$

where $\boldsymbol{R}\left(\boldsymbol{U} ; \boldsymbol{U}_{h}\right)$ stands for the finite residual,

$$
\boldsymbol{R}\left(\boldsymbol{U} ; \boldsymbol{U}_{h}\right)=\boldsymbol{F}-\boldsymbol{A}_{0}(\boldsymbol{U}) \partial_{t} \boldsymbol{U}_{h}-\mathcal{L}\left(\boldsymbol{U} ; \boldsymbol{U}_{h}\right) .
$$

In this work we define the space where the subscales belong as the orthogonal space to the finite element space, $\widetilde{\boldsymbol{W}}=\boldsymbol{W}_{h}^{\perp}$. This is the so called Orthogonal Sub-Grid Scales (OSGS) method, which defines the projection as the orthogonal projection onto the finite element space $\widetilde{\boldsymbol{P}}=\boldsymbol{P}_{h}^{\perp}=\boldsymbol{I}-\boldsymbol{P}_{h}$, being $\boldsymbol{P}_{h}$ the $L^{2}$-projection onto the finite element space. Apart from the construction of the spaces where the subscales belong, we call the subscales dynamic because the temporal derivative of subscales in (18) and (26) is taken into account, and non-linear, as the subscales are accounted for in all the non-linear terms of both the finite scale and subscale equations. This means that at all instances where $\boldsymbol{U}$ appears, it is replaced by $\boldsymbol{U}_{h}+\widetilde{\boldsymbol{U}}$

We also adopt in the present formulation a diagonal matrix of stabilization parameters $\boldsymbol{\tau}^{-1}(\boldsymbol{U})=\operatorname{diag}\left(\tau_{c}^{-1}(\boldsymbol{U}), \tau_{m}^{-1}(\boldsymbol{U}) \boldsymbol{I}, \tau_{e}^{-1}(\boldsymbol{U})\right)$, such that an approximation of the nonlinear operator applied to the subscales is made in each element. The definition for the diagonal matrix of stabilization parameters arises from the perspective of a Fourier analysis, as demonstrated in $[26,29]$. These components are defined for each formulation as

$$
\begin{aligned}
& \text { Conservative variables Primitive variables } \\
& \tau_{c}^{-1}(\boldsymbol{U})=C_{2}(|\boldsymbol{u}|+c) / h \quad, \quad \tau_{c}^{-1}(\boldsymbol{U})=\rho \tau_{m} / h^{2}, \\
& \tau_{m}^{-1}(\boldsymbol{U})=\frac{C_{1} \nu}{h^{2}}+\frac{C_{2}(|\boldsymbol{u}|+c)}{h} \quad, \quad \tau_{m}^{-1}(\boldsymbol{U})=\frac{C_{1} \mu}{h^{2}}+\frac{C_{2} \rho u^{\star}}{h}, \\
& \tau_{e}^{-1}(\boldsymbol{U})=\frac{C_{1} \alpha}{h^{2}}+\frac{C_{2}(|\boldsymbol{u}|+c)}{h} \quad, \quad \tau_{e}^{-1}(\boldsymbol{U})=\frac{C_{1} \lambda}{h^{2}}+\frac{C_{2} \rho c_{v} u^{\star}}{h} \text {. }
\end{aligned}
$$

In these expressions $C_{1}$ and $C_{2}$ are algorithmic parameters that we take as $C_{1}=$ $12 p^{2}$ and $C_{2}=2 p$, where $p$ is the order of the finite element interpolation (not to be confused with the pressure). We also define the kinematic viscosity as $\nu=\mu / \rho$, and the thermal diffusivity as $\alpha=\lambda / \rho c_{p}$. For the primitive variables formulation, we follow the definition in [29] for the matrix of stabilization parameters, in which $u^{\star}$ is a modified velocity that is calculated with the Gauss error function, $u^{\star}=|\boldsymbol{u}|+\operatorname{erf}(\phi) c$, where $\phi$ 
is a normalized compressibility that is defined as $\phi=2-2(\epsilon-M) / \epsilon$. In the previous expression $\epsilon$ is a parameter that determines a certain transition from the compressible to the incompressible regime, which we assume as $\epsilon=0.1$ in the numerical examples presented in this work. Note that for the primitive variables formulation, the stabilization parameter for the momentum equation is dimensionally as the one for the conservative variables multiplied by the density. This is also the case of the stabilization parameter for the energy equation, which is dimensionally as the one for the conservative variables multiplied by $\rho c_{p}$.

\subsubsection{Subscales at the element boundaries}

On the element boundaries, the subscales are calculated as follows. The main idea, proposed originally in [27], is to use the fact that the traction is continuous across element interfaces. The weak continuity of the total fluxes implies that

$$
\sum_{K}\left(\boldsymbol{V}_{h}, n_{k} \boldsymbol{K}_{k j}(\boldsymbol{U}) \partial_{j} \boldsymbol{U}\right)_{\partial K}=0 .
$$

Suppose two elements $K_{1}, K_{2}$ that share an edge (face, in $d=3$ ) $E$. The jump operator of a scalar function $g$ across $E$ is defined as

$$
\llbracket \boldsymbol{n} g \rrbracket_{E}=\left.\boldsymbol{n}^{(1)} g\right|_{\partial K_{1} \cap E}+\left.\boldsymbol{n}^{(2)} g\right|_{\partial K_{2} \cap E},
$$

where $\boldsymbol{n}^{(1)}$ is the unit external normal to element $K_{1}$, and $\boldsymbol{n}^{(2)}$ is the unit external normal to element $K_{2}$. Therefore, the continuity of the fluxes can be imposed with the jump operator on each edge as

$$
0=\llbracket n_{k} \boldsymbol{K}_{k j}(\boldsymbol{U}) \partial_{j} \boldsymbol{U}_{h} \rrbracket_{E}+\llbracket n_{k} \boldsymbol{K}_{k j}(\boldsymbol{U}) \partial_{j} \tilde{\boldsymbol{U}} \rrbracket_{E} .
$$

We can write the second term on the RHS of the previous expression in the following manner:

$$
\llbracket n_{k} \boldsymbol{K}_{k j}(\boldsymbol{U}) \partial_{j} \widetilde{\boldsymbol{U}} \rrbracket_{E}=\mathcal{F}_{\partial K_{1} \cap E}^{(1)}+\mathcal{F}_{\partial K_{2} \cap E}^{(2)}
$$

The approximation of the method is given by the supposition that the fluxes related to the subscales at the boundary $\widetilde{\boldsymbol{U}}_{E}$ are calculated respectively as

$$
\mathcal{F}_{\partial K_{1} \cap E}^{(1)} \approx \mathcal{K} \frac{\widetilde{\boldsymbol{U}}_{E}-\widetilde{\boldsymbol{U}}^{(1)}}{\delta}, \quad \text { and } \quad \mathcal{F}_{\partial K_{2} \cap E}^{(2)} \approx \mathcal{K} \frac{\widetilde{\boldsymbol{U}}_{E}-\widetilde{\boldsymbol{U}}^{(2)}}{\delta} .
$$

Here we approximate the subscales $\widetilde{\boldsymbol{U}}^{(i)}$ at the interior of element $i$, up to a distance $\delta=\delta_{0} h$ to the element boundary, $0 \leq \delta_{0} \leq 1 / 2$. We may introduce $\mathcal{K}$ as an approximation for the diffusion matrix $\boldsymbol{K}_{i j}(\boldsymbol{U})$, for $1 \leq i, j \leq d$, which we define in this work for the conservative and primitive formulations, respectively as

$$
\mathcal{K}=\left[\begin{array}{ccc}
0 & \mathbf{0}^{\top} & 0 \\
\mathbf{0} & \nu \boldsymbol{I} & \mathbf{0} \\
0 & \mathbf{0}^{\top} & \alpha
\end{array}\right] \quad \text { and } \quad \mathcal{K}=\left[\begin{array}{ccc}
0 & \mathbf{0}^{\top} & 0 \\
\mathbf{0} & \mu \boldsymbol{I} & \mathbf{0} \\
0 & \mathbf{0}^{\top} & \lambda
\end{array}\right]
$$

Nevertheless, other types of definitions for the subscales fluxes could be implemented, as finite-difference-like methods. 
With the previous considerations, we obtain a definition for the subscales at the internal edges of the finite element mesh

$$
\tilde{\boldsymbol{U}}_{E}=\{\tilde{\boldsymbol{U}}\}_{E}-\mathcal{K}^{-1} \frac{\delta}{2} \llbracket n_{k} \boldsymbol{K}_{k j}(\boldsymbol{U}) \partial_{j} \boldsymbol{U}_{h} \rrbracket_{E},
$$

where $\{\widetilde{\boldsymbol{U}}\}_{E}$ stands for $\{\widetilde{\boldsymbol{U}}\}_{E}=\left(\widetilde{\boldsymbol{U}}^{(1)}+\widetilde{\boldsymbol{U}}^{(2)}\right) / 2$. As explained in [27], we can neglect the contribution of this term, so that the calculation of the subscales at the edges is given by

$$
\widetilde{\boldsymbol{U}}_{E}=-\boldsymbol{\tau}_{E} \llbracket n_{k} \boldsymbol{K}_{k j}(\boldsymbol{U}) \partial_{j} \boldsymbol{U}_{h} \rrbracket_{E}
$$

The previous equation can be seen as the usual definition for the subscales calculation, this is, to use a boundary matrix of stabilization parameters which accounts for $\tau_{E}=$ $\mathcal{K}^{-1} \delta_{0} h / 2$. In this work, the algorithmic constant $\delta_{0}=1 / 2$ is used.

\subsection{Time integration}

We partition the time interval $\left(0, t_{f}\right)$ in a sequence of discrete time steps $0=t^{0}<$ $t^{1}<\ldots<t^{N}=t_{f}$, with $\delta t>0$ the time step-size defining $t^{n+1}=t^{n}+\delta t$ for $n=$ $0,1,2, \ldots, N$. We implement an implicit monolithic time integration scheme in order to integrate the time derivatives of Eqs. (16) and (26). More specifically, we use the Backward Differentiation Formula (BDF) scheme. For the time dependent function $y(t)$, the BDF approximation of order $k=1,2, .$. , is given by $\delta_{k} y^{n+1} / \delta t$, with

$$
\delta_{k} y^{n+1}=\frac{1}{\gamma_{k}}\left(y^{n+1}-\sum_{i=0}^{k-1} \phi_{k}^{i} y^{n-1}\right)
$$

where $\gamma_{k}$ and $\phi_{k}^{i}$ are numerical parameters. In particular, we use the first order scheme for discretizing the transient term of the subscales in Eq. (26). The solution of the subscales in the interior of the element at the time step $n+1$ is computed from

$$
\widetilde{\boldsymbol{U}}^{n+1}=\boldsymbol{\tau}_{t}\left(\boldsymbol{U}^{n+1}\right)\left(\widetilde{\boldsymbol{P}}\left[\boldsymbol{R}\left(\boldsymbol{U}^{n+1} ; \boldsymbol{U}_{h}^{n+1}\right)\right]+\boldsymbol{A}_{0}\left(\boldsymbol{U}^{n+1}\right) \frac{\widetilde{\boldsymbol{U}}^{n}}{\delta t}\right),
$$

where the dynamic operator $\boldsymbol{\tau}_{t}\left(\boldsymbol{U}^{n+1}\right)$ is defined as

$$
\boldsymbol{\tau}_{t}\left(\boldsymbol{U}^{n+1}\right)=\left(\frac{1}{\delta t} \boldsymbol{A}_{0}\left(\boldsymbol{U}^{n+1}\right)+\boldsymbol{\tau}^{-1}\left(\boldsymbol{U}^{n+1}\right)\right)^{-1}
$$

In the case of the primitive variables formulation, we avoid the off-diagonal terms that appear in matrix $\boldsymbol{A}_{0}\left(\boldsymbol{U}^{n+1}\right)$ by approximating (36) as described in [29]. Hence, for the primitive variables formulation we take the following diagonal definition of the dynamic operator:

$$
\boldsymbol{\tau}_{t}\left(\boldsymbol{U}^{n+1}\right)=\operatorname{diag}\left(\left(\frac{\rho^{n+1}}{p^{n+1} \delta t}+\tau_{c}^{-1}\right)^{-1},\left(\frac{\rho^{n+1}}{\delta t}+\tau_{m}^{-1}\right)^{-1} \boldsymbol{I},\left(\frac{\rho^{n+1} c_{p}}{\delta t}+\tau_{e}^{-1}\right)^{-1}\right) .
$$




\section{Variational subscales as error estimator}

The stabilized variational formulation for the compressible problem has been presented in the previous section. Regarding the discrete finite element approximation, it is identified with the coarse scales, while the variational subscales are related to the solution error. In this section, the error estimation (computed from the variational subscales) is presented. First, we introduce some additional approximations for calculating the subscales at the boundaries of the element. At the end of the section, we present the norms in which the error is measured.

\subsection{Approximation for the subscales at the element boundaries}

The equation for the subscales at the element interior (26) is a non-linear ordinary differential equation that is typically solved at each integration point inside the element. This follows from the fact that subscales need to be included in the finite element scales (23) at the integration points. Nevertheless, the subscales (as error estimation) can be calculated at any location in the interior of the element (e.g. at the center of the element and/or at the nodes). Instead, the subscales at the element boundaries (34) are defined by the inter-element jump operator, and therefore, the contribution of neighbor elements must be accounted for. This type of calculation is inconvenient for parallel implementations of the AMR strategy, in which neighboring elements may be located on a different partition of the computational domain. To overcome this difficulty, we follow the approach in [23] for approximating the calculation of the subscales at the element boundaries: the inter-element jump can be bounded by an orthogonal projection in the interior of the element.

Let us first explain how the boundary subscales are developed. Expanding (34) and writing it for each equation of the compressible problem, results in

$$
\begin{aligned}
\widetilde{\boldsymbol{m}}_{E} & =-\frac{\delta_{0} h}{2}\left(\nu^{-1} \boldsymbol{I}\right) \llbracket \boldsymbol{\sigma}\left(\boldsymbol{U}_{h}\right) \cdot \boldsymbol{n} \rrbracket_{E}, & & \widetilde{\boldsymbol{u}}_{E}=-\frac{\delta_{0} h}{2}\left(\mu^{-1} \boldsymbol{I}\right) \llbracket \boldsymbol{\sigma}\left(\boldsymbol{U}_{h}\right) \cdot \boldsymbol{n} \rrbracket_{E}, \\
\widetilde{e}_{\mathrm{tot}, E} & =\frac{\delta_{0} h}{2}\left(\alpha^{-1}\right) \llbracket \boldsymbol{q}\left(\boldsymbol{U}_{h}\right) \cdot \boldsymbol{n} \rrbracket_{E}, & & \widetilde{T}_{E}=\frac{\delta_{0} h}{2}\left(\lambda^{-1}\right) \llbracket \boldsymbol{q}\left(\boldsymbol{U}_{h}\right) \cdot \boldsymbol{n} \rrbracket_{E},
\end{aligned}
$$

for the conservative and primitive variables, respectively. Note that no subscales over the boundaries result in the case of the continuity equation.

As described before, we approximate the jump of the fluxes. It has been shown in [30] that any jump at the boundaries of the elements possesses a lower, and an upper bound, in the sense that

$$
\begin{aligned}
\gamma_{1} \sum_{K} h_{K} \int_{\partial K}\left|\llbracket \boldsymbol{\sigma}\left(\boldsymbol{U}_{h}\right) \cdot \boldsymbol{n} \rrbracket_{E}\right|^{2} & \leq\left\|\boldsymbol{\sigma}\left(\boldsymbol{U}_{h}\right)-P_{h}\left(\boldsymbol{\sigma}\left(\boldsymbol{U}_{h}\right)\right)\right\|^{2} \\
& \leq \gamma_{2} \sum_{K} h_{K} \int_{\partial K}\left|\llbracket \boldsymbol{\sigma}\left(\boldsymbol{U}_{h}\right) \cdot \boldsymbol{n} \rrbracket_{E}\right|^{2},
\end{aligned}
$$

for certain $\gamma_{1}$ and $\gamma_{2}$. This introduces the possibility of approximating the $L^{2}$-norm of the jumps at the boundaries with

$$
\int_{\partial K}\left|\llbracket \boldsymbol{\sigma}\left(\boldsymbol{U}_{h}\right) \cdot \boldsymbol{n} \rrbracket_{E}\right|^{2} \approx \gamma h_{K}^{-1}\left\|\boldsymbol{\sigma}\left(\boldsymbol{U}_{h}\right)-P_{h}\left(\boldsymbol{\sigma}\left(\boldsymbol{U}_{h}\right)\right)\right\|_{K}^{2} .
$$


A similar result is obtained for the jump over the heat flux:

$$
\int_{\partial K}\left|\llbracket \boldsymbol{q}\left(\boldsymbol{U}_{h}\right) \cdot \boldsymbol{n} \rrbracket_{E}\right|^{2} \approx \gamma h_{K}^{-1}\left\|\boldsymbol{q}\left(\boldsymbol{U}_{h}\right)-P_{h}\left(\boldsymbol{q}\left(\boldsymbol{U}_{h}\right)\right)\right\|_{K}^{2} .
$$

In these approximations, $\gamma$ is a constant that can be calibrated (not to be confused with the ratio of specific heats). Another way to see this approximation is that we are replacing the boundary integral terms by a Zienkiewicz-Zhu estimator [31] over stresses and heat fluxes.

\subsection{Error estimator norms}

Providing a measure of the error for the finite element solution of all variables of the compressible problem is not trivial. Because the equations are neither non-symmetric nor positive definite, there is not a single energy norm (which exists in other fluid flow problems) that could give an estimate of the solution error. Moreover, as we have subscales for each of the equations of the problem, we must define an appropriate norm for estimating the error that includes the contribution of each of the subscales. In the following, we mention two different norms that we develop to compute an error estimator that can be used in AMR. It is obvious that many other alternatives would be possible.

\subsubsection{Scaled $L^{2}$-norm}

One possibility is to define a scaled $L^{2}$-norm of the subscales of the type $|\widetilde{\boldsymbol{U}}|_{S}^{2}=$ $\widetilde{\boldsymbol{U}}^{\top} \boldsymbol{S} \widetilde{\boldsymbol{U}}$. The scaling matrix $\boldsymbol{S}$ is intended to guarantee the dimensional consistency between the different subscales. This scaled norm can be applied considering the interior subscales and the subscales at the element boundaries, such that the error estimator at each element is computed as

$$
\eta_{K}^{2}:=\int_{K}|\widetilde{\boldsymbol{U}}|_{S}^{2}+\int_{\partial K}\left|\widetilde{\boldsymbol{U}}_{E}\right|_{S}^{2}
$$

where it is understood that $\widetilde{\boldsymbol{U}}$ is restricted to $K$ (and $E \in \partial K$ ). The scaling matrices for each of the subscales in the previous equation may be different. In this work, we adopt the diagonal matrix of stabilization parameters $\tau$ as part of the scaling matrix, and apply the same scaling matrix definition for both $\widetilde{\boldsymbol{U}}$ and $\widetilde{\boldsymbol{U}}_{E}$.

Introducing a reference velocity of the fluid flow problem $u_{0}=\left|\boldsymbol{u}_{h}\right|+c$, we can take for the conservative variables formulation a scaling matrix $\boldsymbol{S}=\operatorname{diag}\left(\tau_{c}^{-1} u_{0}^{2}, \tau_{m}^{-1} \boldsymbol{I}, \tau_{e}^{-1} u_{0}^{-2}\right)$, that gives the following dimensionally-consistent estimation of the error at each element:

$$
\begin{aligned}
\eta_{K}^{2}:= & \int_{K} \tau_{c}^{-1}\left(\left|\boldsymbol{u}_{h}\right|+c\right)^{2}|\widetilde{\rho}|^{2}+\int_{K} \tau_{m}^{-1}|\widetilde{\boldsymbol{m}}|^{2}+\int_{K} \tau_{e}^{-1}\left(\left|\boldsymbol{u}_{h}\right|+c\right)^{-2}\left|\widetilde{e}_{\mathrm{tot}}\right|^{2} \\
& +\int_{\partial K} \tau_{m, E}^{-1}\left|\widetilde{\boldsymbol{m}}_{E}\right|^{2}+\int_{\partial K} \tau_{e, E}^{-1}\left(\left|\boldsymbol{u}_{h}\right|+c\right)^{-2}\left|\widetilde{e}_{\mathrm{tot}, E}\right|^{2} .
\end{aligned}
$$

In the case of the primitive variables formulation, the scaling matrix $\boldsymbol{S}$ can be written as $\boldsymbol{S}=\operatorname{diag}\left(\tau_{c}^{-1} \rho_{h}^{-1}, \tau_{m}^{-1} \boldsymbol{I}, \tau_{e}^{-1} T_{h}^{-1}\right)$, being $\rho_{h}$ and $T_{h}$ the finite element approximation of the density and temperature, respectively. Therefore, in the case of the primitive 
formulation the error estimation at each element is given by the following dimensionallyconsistent norm:

$$
\begin{aligned}
\eta_{K}^{2}:= & \int_{K} \tau_{c}^{-1} \rho_{h}^{-1}|\widetilde{p}|^{2}+\int_{K} \tau_{m}^{-1}|\widetilde{\boldsymbol{u}}|^{2}+\int_{K} \tau_{e}^{-1} T_{h}^{-1}|\widetilde{T}|^{2} \\
& +\int_{\partial K} \tau_{m, E}^{-1}\left|\widetilde{\boldsymbol{u}}_{E}\right|^{2}+\int_{\partial K} \tau_{e, E}^{-1} T_{h}^{-1}\left|\widetilde{T}_{E}\right|^{2} .
\end{aligned}
$$

\subsubsection{Entropy measure}

Another possibility is to construct the error measure based on the calculation of an entropy functional. Assuming that the fluid possesses a constant specific heat at constant volume, the fundamental equation for the perfect gas is

$$
s=s_{0}+c_{v} \ln \left(\frac{e}{e_{0}}\right)-R \ln \left(\frac{\rho}{\rho_{0}}\right),
$$

where $s_{0}, e_{0}$, and $\rho_{0}$ are reference values of entropy, internal energy, and density, respectively. Since the perfect gas relation can be written as $p=\rho(\gamma-1) e$, and the caloric equation relates the internal energy with temperature, the previous relation can be developed as

$$
s=s_{0}+c_{v} \ln \left(\frac{p}{\rho^{\gamma}}\right)+c_{v} \ln \left(\frac{\rho_{0}^{\gamma-1}}{(\gamma-1)}\right)-c_{v} \ln e_{0} .
$$

Therefore, an entropy function can be defined for the compressible problem as

$$
s=c_{v} \ln \left(\frac{p}{\rho^{\gamma}}\right)+\hat{s}_{0},
$$

with $\hat{s}_{0}$ denoting the coefficients of (45) which are reference quantities. Since calculating an entropy with the subscales alone may lead to unphysical results, the entropy is used as an estimator by calculating the elemental relative $L^{2}$-error between the entropy of the finite solution including the subscales $\widetilde{s}$, and the entropy of the finite solution alone $s_{h}$. This is,

$$
\eta_{K}^{2}:=\int_{K} \frac{\left(s\left(\boldsymbol{U}_{h}\right)-s\left(\boldsymbol{U}_{h}+\widetilde{\boldsymbol{U}}\right)\right)^{2}}{s\left(\boldsymbol{U}_{h}\right)^{2}}
$$

For the conservative variables formulation, we calculate the entropy as

$$
s(\boldsymbol{U})=c_{v} \ln \left(\frac{(\gamma-1)\left(e_{\mathrm{tot}}-\frac{|\boldsymbol{m}|^{2}}{2 \rho}\right)}{\rho^{\gamma}}\right) .
$$

Similarly, for the primitive variables formulation, the entropy is calculated as

$$
s(\boldsymbol{U})=c_{v} \ln \left(\frac{p}{\left(\frac{p}{R T}\right)^{\gamma}}\right) .
$$

Note that, while the scaled $L^{2}$-norm gives an error estimation that has the dimensions of the scaled subscales, the relative $L^{2}$-error of the entropy leads to a non-dimensional error estimation. This difference between the entropy meassure and the scaled $L^{2}$-norm has consequences in the AMR, as it will be explained in the numerical examples. 


\section{Numerical Examples}

The error estimator presented before is now tested in AMR simulations of compressible flow problems. Two steady exact solution examples are solved in the first part of this section. The simulation of exact solution problems is mainly devoted to highlighting the behavior of the subscales as explicit error estimators. Then, the three-dimensional lid-driven cavity and the unsteady differentially heated cavity examples are intended to study the error estimation in subsonic flow problems. The primitive variables formulation is used in these cases, since this formulation simplifies to the incompressible equations when the incompressible constraint is included, and consequently, it is well defined in the low Mach number limit. On the contrary, the conservative variables formulation admits physically meaningful solutions when the solution develops discontinuities, as in the case of supersonic shocks, and consequently, we use that formulation to solve the last two numerical examples: the flow over a flat plate and the flow past a cylinder supersonic flow problems. In the case of those supersonic flow examples, the conservative variables formulation is enhanced with the orthogonal projection based shock capturing and the anisotropic imposition of the added numerical diffusivity introduced in [26].

As described in Section 3, we implement an implicit monolithic time integration scheme in order to integrate the time derivatives of Eqs. (16) and (26). More specifically, we use the second order accurate Backward Differentiation Formula (BDF) scheme, so that, at each time step we solve the non-linearities of both the finite element and subgrid scales by using Picard's scheme. This leads to a monotonically decreasing relative error between consecutive iterations, with the subsequent convergence of the numerical method. At most ten iterations are performed, fulfilling the maximum relative numerical tolerance for the $L^{2}$ norm iteration residual of $10^{-10}$.

As commented before, the refinement process is held locally to counterbalance the error through the computational domain. The basic idea is, to begin with a given ( 0 -level) coarse grid, and then to subsequently perform refinement (or coarsening). After each time step of the transient problem is solved we estimate the local error using (41) or (47). The error estimation is used to refine or coarsen the elements of the mesh depending on a given tolerance criteria (or threshold). The algorithm for refining the mesh is based on the h-adaptivity method of RefficientLib [28] that splits the element into sub-elements, or removes sub-elements to coarsen the mesh (being the 0-level mesh the coarsest possible mesh). We advance in time and adapt the mesh until a temporal convergence criterion is satisfied. In the case of non-transient examples, we perform the refinement step after each Picard's iteration. From now onwards the flow is considered as an ideal gas, with ratio of specific heats $\gamma=1.4$ and physical properties $c_{p}=1.010 \mathrm{~kJ} /(\mathrm{kg} \mathrm{K})$ and $c_{v}=0.718 \mathrm{~kJ} /(\mathrm{kg} \mathrm{K})$.

\subsection{Smooth exact solution}

The first numerical example is intended to study the ability of the subscales to act as an explicit error estimation. In this case, we evaluate the estimation of the error given by the subscales, specifically, we consider the error measured with the scaled $L^{2}$-norm of an exact solution problem. The exact solution problem is solved by calculating an exact force, computed with the residual of the continuous problem, that is used in the discrete problem (also known as the method of manufactured solutions). 
We use the primitive variables formulation to solve this numerical example. The problem is defined inside a rectangular domain $[0, L] \times[0, L]$, with $L=1 \mathrm{~m}$, with the viscosity fixed to $\mu=0.1 \mathrm{~kg} /(\mathrm{m} \mathrm{s})$, and the thermal conductivity to $\lambda=1000 \mathrm{~W} /(\mathrm{m}$ $\mathrm{K})$. The polynomial functions that we use as a steady two-dimensional manufactured solution for the compressible Navier-Stokes equations are given as follows:

$$
\begin{aligned}
p & =x_{1}^{2} x_{2}^{2}\left(x_{1}-1\right)\left(x_{2}-1\right)+p_{\text {atm }}, \\
u_{1} & =2 x_{1}^{2} x_{2}\left(x_{1}-1\right)^{2}\left(x_{2}-1\right)\left(2 x_{2}-1\right), \\
u_{2} & =-2 x_{1} x_{2}^{2}\left(x_{1}-1\right)\left(x_{2}-1\right)^{2}\left(2 x_{1}-1\right), \\
T & =2 x_{1}^{2} x_{2}\left(x_{1}^{2}-1\right)\left(x_{2}-1\right)+T_{\text {atm }} .
\end{aligned}
$$

Reference values for the temperature and pressure variables are fixed as $T_{\text {atm }}=0.01 \mathrm{~K}$ and $p_{\text {atm }}=0.01 \mathrm{~Pa}$, respectively.

This type of problem is favorable to analyze the error estimation. Indeed, the difference between the discrete solution and the exact solution can be calculated, and it can be compared to the error given by the subscales. In this numerical example, we restrain the analysis to the scaled $L^{2}$-norm (presented in Section 4), as a measure of the subscales-based error, and we also use this norm to calculate the error against the exact solution. We prescribe the tolerance below the subscales-based error estimation through the simulation, so that, the algorithm refines uniformly the mesh.

This example is used to adjust the $\gamma$ parameter of approximation (38), which is made to compute the integral of the subscales at the element boundaries. To this end, we perform different homogeneous refinement simulations varying the $\gamma$ parameter and evaluate the efficiency of each subscales-based error estimation. Figure 1 displays the error convergence of the exact error compared with the subscales-based error estimator for the different values of $\gamma$. The efficiency index of the subscales-based estimator is also presented on the right side of this figure. In the convergence plot, we see that neglecting the subscales at the boundary $(\gamma=0)$ underestimates the error. Fixing $\gamma=0.1$ gives the most adjusted error estimation in contrast to the exact error. It can be observed that the efficiency index of the estimator for this value is very good. The case of having $\gamma>0.1$ overestimates the error, which is also observed in the detached result for the efficiency index. Hence, we fix $\gamma=0.1$ to approximate the integral of the subscales over the boundary of the element in the following numerical examples.

We also show the spatial distribution of the exact error and the subscales-based error estimator in Fig. 2, after some refinement steps have been done. We see how it properly matches the spatial distribution of the exact error, and thus we validate the ability of the subscales-based error estimator to capture the error associated with the discretization error.

\subsection{Singular exact solution}

The second numerical example is a two-dimensional exact solution comprising a singularity in the solution. This allows us to evaluate the performance of the subscales as an error estimator when large localized gradients appear. We also use the primitive variables formulation to investigate the separated contribution of the subscales associated to each equation of the compressible problem into the estimation of the error. The exact 

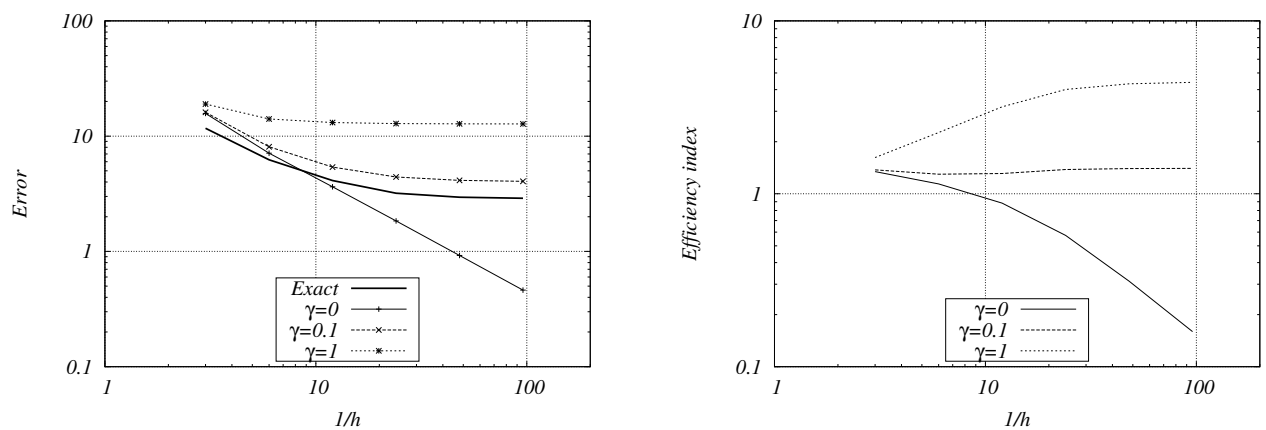

Figure 1: Smooth exact solution results: convergence of the exact error and the subscales error estimator at the left, and efficiency index as a function of the mesh size at the right.
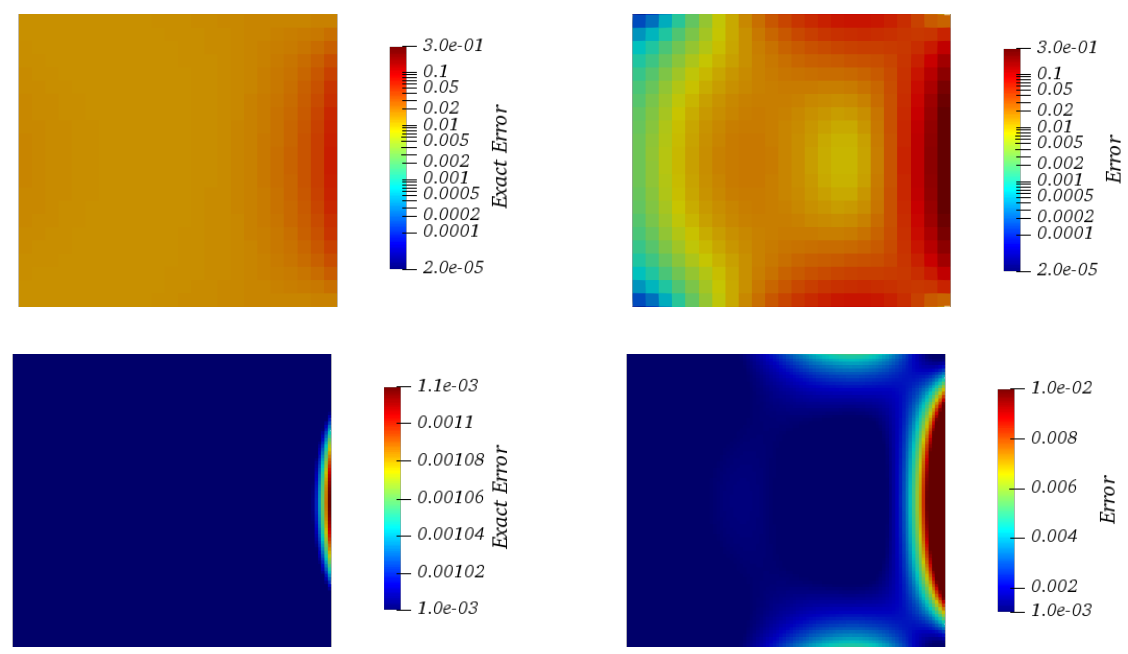

Figure 2: Smooth exact solution results: scaled $L^{2}-$ norm of the exact error at the left, and scaled $L^{2}$-norm of the subscales at the right. Top: after three refinements. Bottom: after five refinements. 
solution is evaluated in the $L$-shaped domain $([-1,1] \times[-1,1]) \backslash([0,1] \times[-1,0])$, with the following functions:

$$
\begin{aligned}
p(r, \phi) & =-r^{\alpha-1} \frac{(1+\alpha)^{2} \varphi^{\prime}(\phi)+\varphi^{\prime \prime \prime}(\phi)}{1-\alpha}+p_{\text {atm }}, \\
\boldsymbol{u}(r, \phi) & =r^{\alpha}\left[\begin{array}{c}
\cos (\phi) \varphi^{\prime}(\phi)+(1+\alpha) \sin (\phi) \varphi(\phi) \\
\sin (\phi) \varphi^{\prime}(\phi)-(1+\alpha) \sin (\phi) \varphi(\phi)
\end{array}\right], \\
T(r, \phi) & =r^{\alpha}\left(\cos (\phi) \varphi^{\prime}(\phi)+(1+\alpha) \sin (\phi) \varphi(\phi)\right)+T_{\text {atm }},
\end{aligned}
$$

with $r$ and $\phi$ being the polar coordinates, and the function $\varphi$ defined as

$$
\varphi(\phi)=\frac{\sin ((1+\alpha) \phi) \cos (\alpha \omega)}{1+\alpha}-\cos ((1+\alpha) \phi)+\frac{\sin ((1+\alpha) \phi) \cos (\alpha \omega)}{1-\alpha}+\cos ((\alpha-1) \phi) .
$$

Here we take $\omega=3 \pi / 2$ and $\alpha$ as the (approximate) root of the following non-linear equation:

$$
\frac{\sin ^{2}(\alpha \omega)-\alpha^{2} \sin ^{2}(\omega)}{\alpha^{2}}=0
$$

In this case the viscosity fixed to $\mu=0.1 \mathrm{~kg} /(\mathrm{m} \mathrm{s})$, and the thermal conductivity to $\lambda=25700 \mathrm{~W} /(\mathrm{m} \mathrm{K})$. Reference values for the temperature and pressure variables are fixed as $T_{\text {atm }}=1000 \mathrm{~K}$ and $p_{\text {atm }}=10^{5} \mathrm{~Pa}$, respectively.

We first study the comparison between the error estimator and the exact solution error after several refinements steps. We use the scaled $L^{2}$-norm as the measure of the error, and let the mesh adaptation algorithm advance with the tolerance prescribed to $10^{-2}<\eta<10^{-1}$. Figure 3 shows the resulting fields, including the subscales-based error distribution over the refined mesh. The singularity in the pressure field near the corner of the domain can be appreciated at the top of this figure. It can also be seen that the refined mesh especially describes the singular point of the solution.

We also use this numerical example to analyze the error estimation given by the separated subscales (and the consequent type of refinement). Again, we use the scaled $L^{2}$-norm, but we account for the separated contribution of the subscales associated to each equation of the problem. The refined meshes and the subscales-based error measured with the scaled $L^{2}$-norm are displayed in Fig. 4. In this figure, we can see how accounting for the subscales of the mass equation refines the singular point, but the estimated error is high elsewhere. For the momentum subscales, this is not the case, it describes the singular point, but the estimated error is also low in the complete domain. In the case of the subscales of energy, there is not a description of the singularity, yet the estimated error is low.

Another analysis that we make is the comparison between a homogeneous refinement of the mesh and the one driven by the subscales-based error estimator. Figure 5 shows the convergence of the exact error (in the scaled $L^{2}$-norm) against the number of elements. At the left side of this figure, we plot the exact error convergence in the case of the AMR driven by subscales comparison against the homogeneously refined solution. The plot demonstrates the improved convergence of the exact error with the use of the subscales-based error (labeled as $p \boldsymbol{u} T$ ) in contrast to the homogeneous refinement, which 
is not able to accurately represent the singularity. Indeed, the exact error for the AMR converges below the homogeneous refinement at a smaller number of elements in the mesh, from which we conclude that the subscales-based error estimator is suitable for problems in which the solution presents singularities. We also present the computational cost of the subscales-based AMR and of the homogeneous refinement at the right side of Fig. 5. We observe that for the refinement with the subscales-based error estimator the computational effort flattens when the error converges. This is not achievable with the homogeneous refinement, as the computational effort grows with respect to the refinement.

Finally, we test the error estimation given by the separated contribution of the subscales and analyze the error convergence against the number of elements. We perform the refinement of the mesh with the error estimation given by the separated contribution of the subscales (associated with each equation of the problem). The exact error convergence of the different subscale estimations are presented in Fig. 6. We observe that the error diverges when the mass equation subscales (labeled $p$ ) leads the refinement. For overcoming this problem, we see that the subscales of momentum and energy equations are crucial, and therefore, the complete contribution of all subscales in the scaled $L^{2}$-norm improves the convergence of the error. In other words, the subscales of momentum (labeled $\boldsymbol{u}$ in the plot) and energy (labeled $T$ ) contribute the most to the error convergence; as long as these terms are considered both in the interior and on the boundaries of the elements.

\subsection{Three-dimensional lid-driven cavity}

The third case that we solve is the three-dimensional lid-driven cavity. We use this example to compare the scaled $L^{2}$-norm estimation of the subscales-based error with the one given by the entropy measure. The three-dimensional lid-driven cavity problem is defined as a prismatic cavity $[0, L] \times[0, L] \times[0, L]$, with $L=1 \mathrm{~m}$. The flow is initially at rest, with a homogeneous pressure of $0.7124 \mathrm{~Pa}$ and a homogeneous temperature of $0.0024 \mathrm{~K}$. The upper wall $\left(x_{1}, x_{2}, L\right)$ is constantly moving at a fixed velocity of $(1,0,0)$ $\mathrm{m} / \mathrm{s}$. For the upper boundary, the temperature is also set to $0.0024 \mathrm{~K}$. A no-slip condition for velocity, an adiabatic condition for energy, and an impermeable condition for mass are set over the other walls. We set the Prandtl number to $\mathrm{Pr}=0.71$ and the Reynolds number to $\operatorname{Re}=1000$. At this conditions, the compressibility regime of the flow is $M=1$.

The primitive variables formulation is used to solve this numerical example: the resulting flow is laminar and steady; it is obtained by running the simulation until the $L^{2}$-error norm between consecutive temporal results is below the transient converge criterion for all variables of the flow problem. The steady flow results are presented in Fig. 7, where the contours for pressure, velocity magnitude, and temperature, at four different cutting planes of the cavity, are presented. We observe that the laminar flow is comprised of a major vortical structure, with singular points of pressure near the top corners of the cavity.

We execute AMR simulations by fixing the error tolerance below $10^{-6}$ for the scaled $L^{2}$-norm, and $10^{-8}$ for the entropy measure; then we perform several consecutive refinements from an initial structured mesh composed of 35937 hexahedral elements. The final adapted meshes and the error estimation over these meshes are presented in Figures 8 and 9 , for the scaled $L^{2}-$ and entropy measures, respectively. The error estimation, which is below the prescribed tolerance in both cases, is plotted as contours at four different 


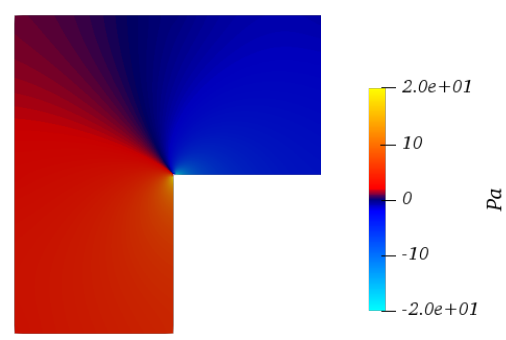

(a)

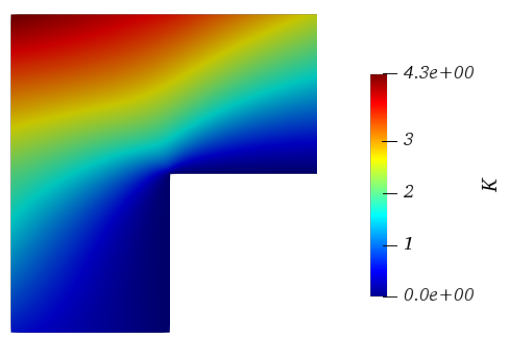

(c)

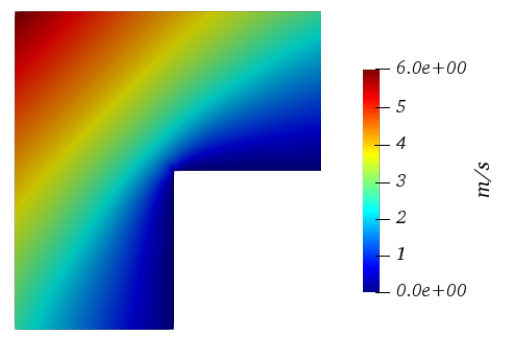

(b)

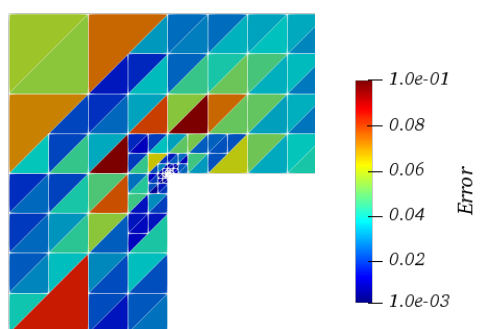

(d)

Figure 3: Singular exact solution results: (a) contour of the relative part of the pressure, (b) contour of the velocity magnitude, (c) contour of the relative part of the temperature, and (d) refined mesh with the subscales-based error distribution. 


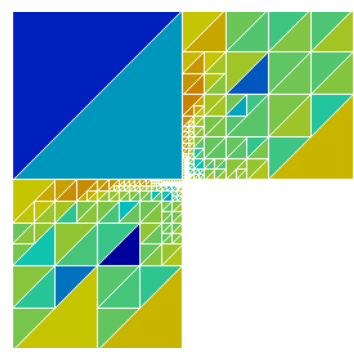

(a)
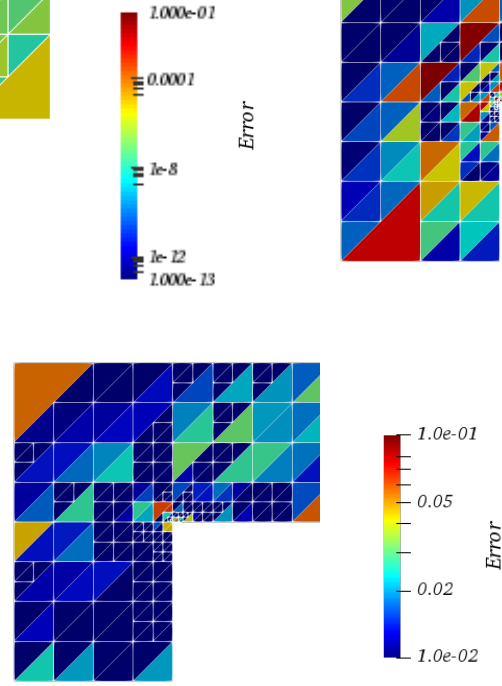

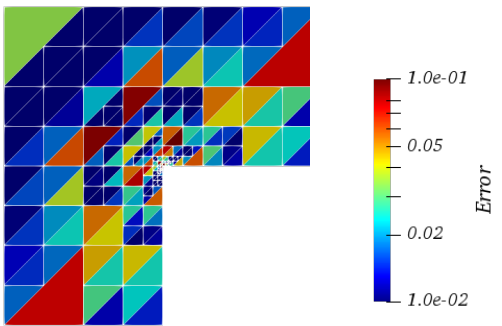

(b)

(c)

Figure 4: Singular exact solution results. Refined mesh and subscales-based error measured with the scaled $L^{2}$-norm considering only the subscales of (a) mass, (b) momentum, and (c) energy.
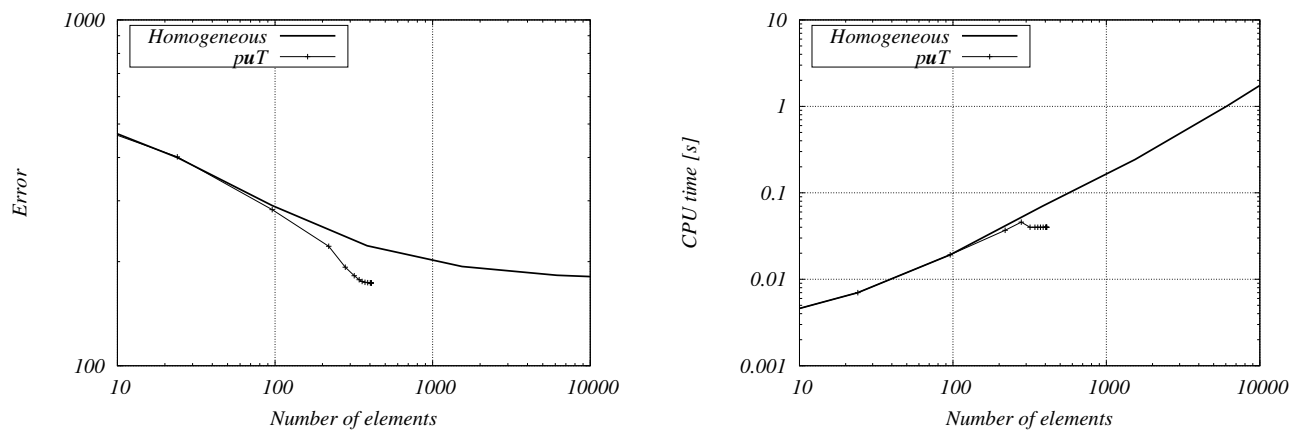

Figure 5: Singular exact solution results: exact error convergence measured with the scaled $L^{2}$-norm against the number of elements at the left, and computational time versus number of elements at the right. 


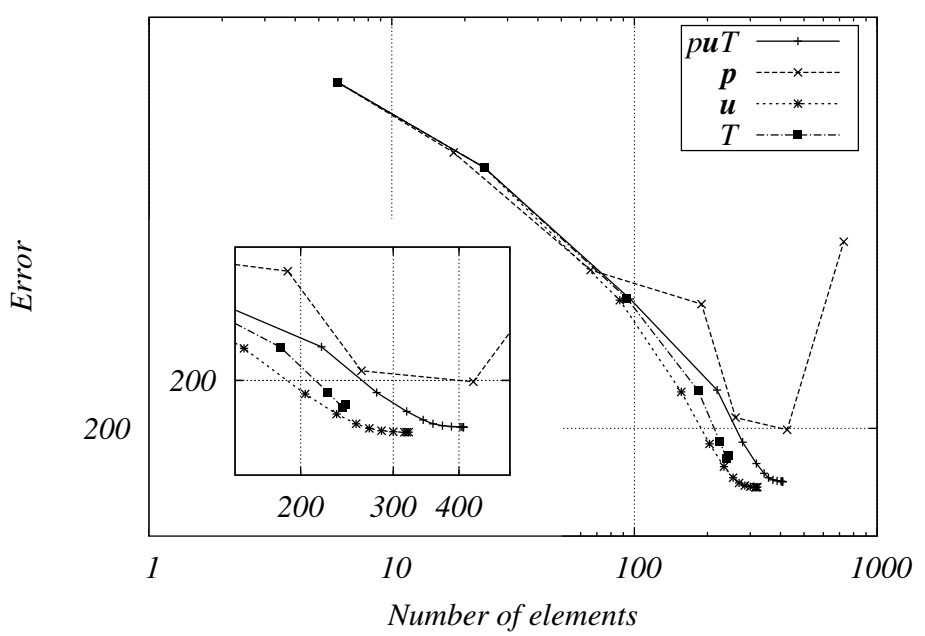

Figure 6: Singular exact solution results. Exact error convergence measured with the scaled $L^{2}-$ norm against the number of elements for the separated subscales refinement.

cutting planes of the cavity. As commented in Section 4, we observe that the measured subscales-based error is smaller in the case of the entropy measure than the one with the scaled $L^{2}$-norm. This is explained as the former in non-dimensional, while the scaled $L^{2}$-norm estimation has the dimensions of the scaled subscales. Hence, the prescribed tolerance can be reduced when the error estimation is measured with the entropy measure; this may lead to a similar number of total elements, as in the case of the scaled $L^{2}$-norm measurement. Either description of the three-dimensional flow pattern given by the subscales-based error estimation is accurate: the resolution of three-dimensional flow singularities, including the description of the boundary layer near the top wall, and the singular points of pressure near the corners, are well identified. This is more evident in the case of the localized refinement that appears at the singular points near the edges and corners, and in the absence of refinement in the regions of the flow where stagnation occurs.

\subsection{Differentially heated cavity}

The fourth case that we study is the differentially heated cavity. In this problem we study the error estimation given by the particular design of the variational subscales, that is, we test the orthogonal, dynamic, and non-linear characteristics of the subscales in an unsteady problem. The flow is considered as a two-dimensional flow confined inside a rectangular cavity $[0, L] \times[0, H]$ of aspect ratio $H / L=8$, with $L=1 \mathrm{~m}$. The temperature at the left (hot) wall is fixed to $T_{H}=600 \mathrm{~K}$, and the temperature at the right (cold) wall to $T_{C}=300 \mathrm{~K}$. No slip and impermeable conditions are set over the cavity walls, together with adiabatic boundary conditions for the upper and lower walls. Gravity is specified to be acting in the negative $x_{2}$ direction as $\boldsymbol{g}=(0,-9.8) \mathrm{m} / \mathrm{s}^{2}$. The initial pressure, temperature, and density conditions for the fluid are $152525 \mathrm{~Pa}, 450 \mathrm{~K}$, and $1.16 \mathrm{~kg} / \mathrm{m}^{3}$, 


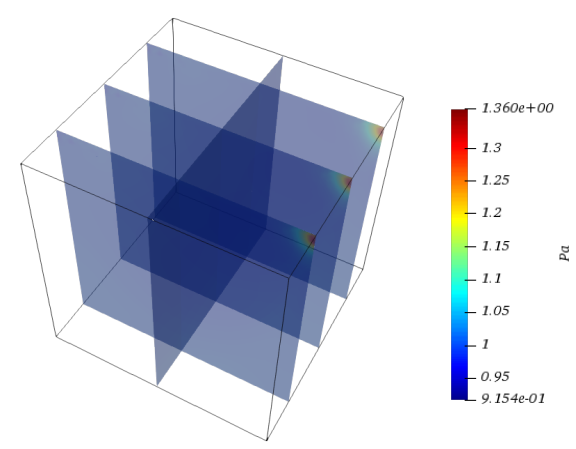

(a)

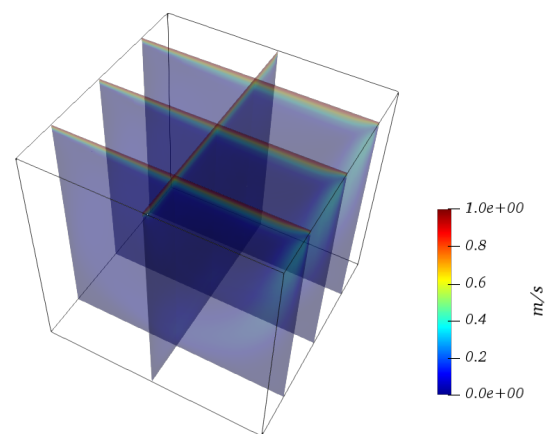

(b)

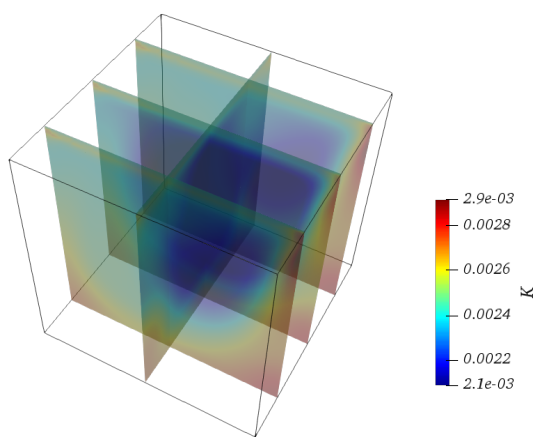

(c)

Figure 7: Three-dimensional lid-driven cavity results: (a) pressure, (b) velocity magnitude, and (c) temperature contours obtained using the refined mesh driven by the subscales-based error measured with the scaled $L^{2}-$ norm.
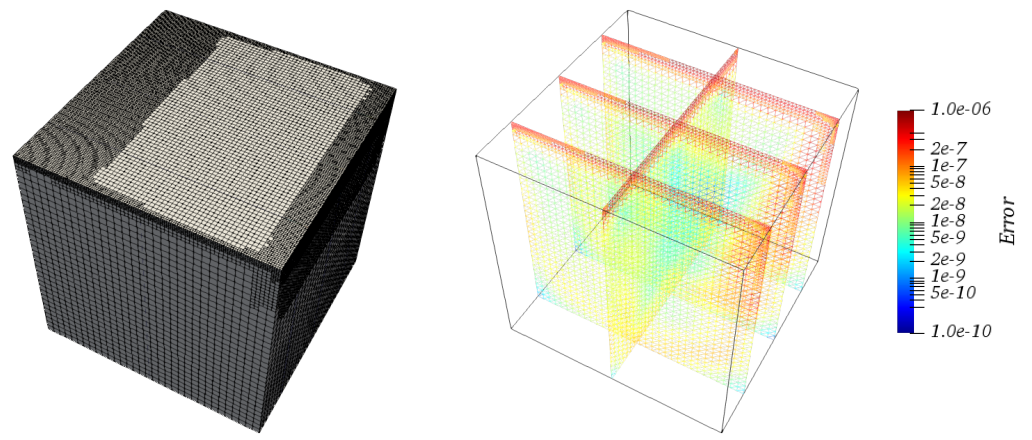

Figure 8: Three-dimensional lid-driven cavity results. Subscales-based error measured with the scaled $L_{2}$-norm: refined mesh composed by 86961 hexahedral elements on the left, and estimated error over four different cutting planes of the refined mesh on the right. 

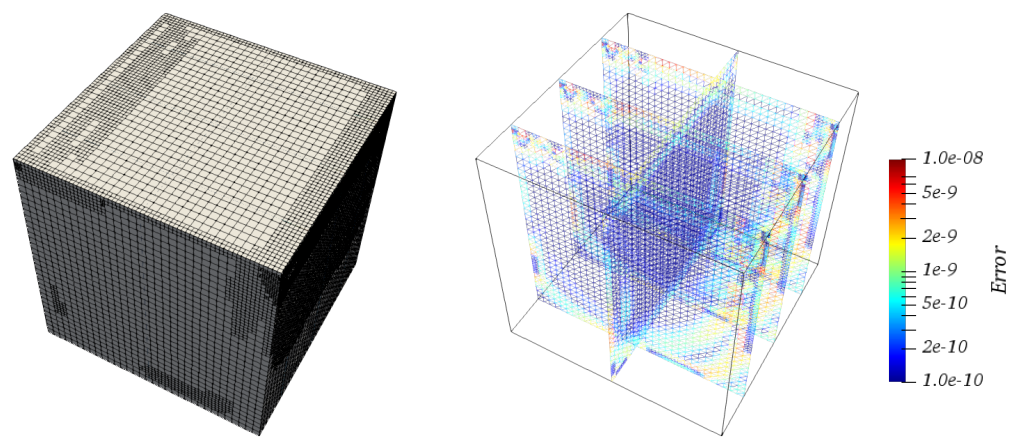

Figure 9: Three-dimensional lid-driven cavity results. Subscales-based error measured with the entropy measure: refined mesh composed by 83634 hexahedral elements on the left, and estimated error over four different cutting planes of the refined mesh on the right.

respectively. The viscosity and thermal conductivity are set to $\mu=2.5 \times 10^{-3} \mathrm{~kg} /(\mathrm{m}$ $\mathrm{s})$, and $\lambda=3.55 \mathrm{~W} /(\mathrm{m} \mathrm{K})$, correspondingly. The non-dimensional Rayleigh number is $\mathrm{Ra}=|\boldsymbol{g}| \theta \rho^{2} c_{p} /(\mu \lambda)=10^{6}$, where $\theta$ stands for the dimensionless temperature ratio $\theta=2\left(T_{H}-T_{C}\right) /\left(T_{H}+T_{C}\right)=0.66$. The simulation is run with a constant time step size of $\delta t=10^{-2} \mathrm{~s}$ until the statistically stationary state (measured as the relative error between consecutive transient results of time-averaged variables) is reached.

In order to overcome the mechanical restriction of the pressure imposition for transient and variable flows at closed computational domains, an iterative penalization to the mass conservation equation, of the form $\left(q_{h}, \psi\left(p_{h}^{* i+1}-p_{h}^{* i}\right)\right)$ at iteration $i+1$, is included in the stabilized formulation. This penalization guarantees that $p_{h}$ is solved correctly, up to a constant, when the relative value of pressure is not set at the computational boundary. The factor $\psi$ is selected numerically as $\psi=10^{-3} \rho / \mu$, in a way that it does not detriment neither the nonlinear scheme convergence (when $\psi$ is large) nor the algebraic solver convergence (when $\psi \rightarrow 0$ ), as discussed in [32].

Moreover, we have observed that including the penalization to the mass conservation equation increases the non-linearity of the stabilized problem: it affects the dynamic subscales approximation since the transient term related to the pressure subscale in (37) is in fact divided by the pressure. Consequently, in this numerical example we modify this dependence by scaling the mass term of the dynamic operator in a different way, we use $\left(\rho^{n+1} h\right) /\left(\mu\left(|\boldsymbol{u}|^{n+1}+c^{n+1}\right) \delta t\right)$ instead of $\rho^{n+1} /\left(p^{n+1} \delta t\right)$.

The transient character of the flow is firstly used for tracking the AMR simulations driven by the subscales-based error. We run the transient simulation using the primitive variables formulation and perform refinements both using the scaled $L^{2}$-norm and the entropy measure at the same two instants of time. The refinement tolerance is fixed to $0.1<\eta_{K}^{2}<1$, in the case of the scaled $L^{2}$-norm, and to $10^{-14}<\eta_{K}^{2}<10^{-10}$, in the case of the entropy measure. The refined meshes and the error distributions are presented in Fig. 10 corresponding to the same instants of time. The temperature contours, including some velocity streamlines, are shown at the left side of the figure. At the center, we display the refined meshes driven by the subscales-based error estimator measured with the scaled $L^{2}-$ norm. The refined meshes driven by the subscales-based error and calculated 
with the entropy measure are plotted at the right side of the figure. It has been reported in [29] that the resolution of flow boundary layers and small perturbations of temperature (producing buoyancy) is enhanced with the inclusion of the dynamic subscales. We also observe that the error distribution, which is below the prescribed tolerance, and the mesh refinement are enhanced mostly at the lateral walls: the subscales-based error estimator is able to describe the boundary layer generated by the buoyancy of the flow. Moreover, the subscales-based error is able to track the relevant variations of the flow, so that, the mesh refinement is attached to the main flow structures through the simulation. It can also be seen that the coarsening of the mesh is carried out when laminar regions of the flow are found.

We can go deeper in the analysis of the transient behavior of the subscales as error estimator by performing calculations of the non-dimensional Nusselt number associated with this problem. Specifically, we investigate, in a qualitative manner, the influence of the subscales design in the estimation of the subscales-based error. For this, we calculate the Nusselt number, which relates the heat transferred from the hot to the cold wall, and which is calculated as

$$
\operatorname{Nusselt}(\boldsymbol{x}, t)=\frac{L}{T_{H}-T_{C}} n_{j} \partial_{j} T(\boldsymbol{x}, t)
$$

over the hot wall of the cavity, and over a time window of $50 \mathrm{~s}$ after the statistically steady state is reached. In particular, the transient behavior of the Nusselt number can be evaluated by integrating the previous equation along the wall and averaging this result in time. We denote the integral result as $\overline{\text { Nusselt }}$, where $\overline{(\cdot)}$ stands for the discrete time average.

A reference value of $\overline{\text { Nusselt }}_{\text {ref }}=52473.40$ is obtained by simulating the problem with an homogeneously refined mesh containing 2323 bi-quadratic elements and the subscales defined as quasi-static and residual-based because this method is similar to the Streamline Upwind Petrov Galerkin (SUPG) method when linear elements are used, and the later has been widely tested in the literature in several compressible flow problems (as in [33]). In the reference simulation, we use the second order accurate BDF as the time integration scheme, and a constant time step size of $\delta t=0.01 \mathrm{~s}$. The obtained reference Nusselt is used for qualitative comparisons: we calculate a (reference) $L^{2}$-error between the Nusselt obtained with the AMR simulations (driven by the subscales-based error) and the reference Nusselt. This reference error is calculated only for comparison reasons, and should not be confused as to be a goal-based error estimator.

We run AMR simulations using the subscales-based error estimator accounting for the different possibilities in the subscales design, and using both the scaled $L^{2}-$ and entropy measures. We evaluate the inclusion of the dynamic subscales against the quasistatic subscales (the ones that neglect the temporal tracking of the subscales), and the orthogonal subscales against the residual subscales (which neglect the projection of the residual into the finite element space).

The time-averaged results of the calculated Nusselt number are presented in Tables 1 and 2. In those tables, we also present the error against the reference Nusselt, and the time-averaged subscales-based error estimation for the different subscales methods. In the case of the scaled $L^{2}$-norm, we find that the subscales-based error estimation matches correctly the reference error; the use of the scaled $L^{2}-$ norm as a measure of the subscales-based error results in the accurate definition of the chaotic behavior, and 


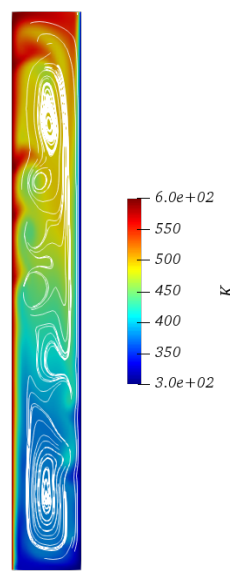

(a)
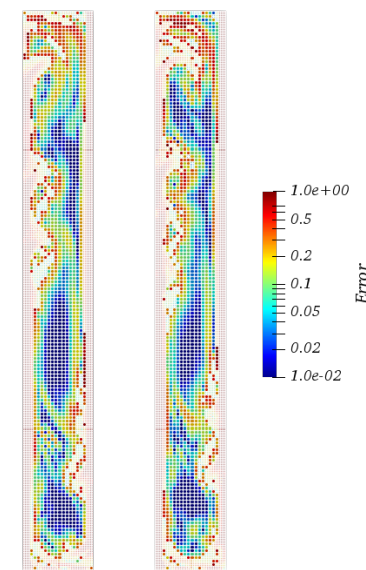

(b)
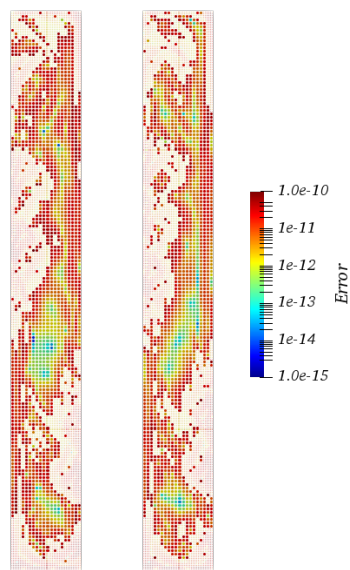

(c)

Figure 10: Diferentially heated cavity results: (a) temperature contours with velocity streamlines, (b) scaled $L^{2}$-norm of the variational subscales: refinement tolerance is fixed $0.1<\eta_{K}^{2}<1$, which led to meshes of 6795 , and 6852 elements, and (c) entropy measure of the variational subscales: refinement tolerance is fixed to $10^{-14}<\eta_{K}^{2}<10^{-10}$, which led to meshes of 6853 , and 7035 elements. Solution is obtained using the primitive formulation and it is presented for the same instants of time.

the estimation of the error made by the subscales provides accurate approximations in comparison with the Nusselt reference error. We also observe that defining the subscales as orthogonal to the finite element space do not improves the approximation, and that, the dynamic definition of the subscales results in the most accurate description of the unsteady character of the flow made by the AMR simulations.

In the case of the subscales-based error measured with the entropy measure, the accuracy of the numerical approximation of the unsteady compressible flow problem matches the estimation of the Nusselt reference error, except when the orthogonal subscales are included. We also observe that the orthogonal subscales do not improve the numerical approximation of the AMR in contrast to the residual subscales, and that, the accuracy of the approximation is improved with the inclusion of the dynamic subscales and the residual definition of the subscales.

Table 1: Differentially heated cavity AMR simulations driven by the subscales-based error and measured with the scaled $L^{2}-$ norm

\begin{tabular}{|l|c|c|c|c|}
\cline { 2 - 5 } \multicolumn{1}{c|}{} & \multicolumn{2}{c|}{ Residual } & \multicolumn{2}{c|}{ Orthogonal } \\
\cline { 2 - 5 } \multicolumn{1}{c|}{} & Quasi-static & Dynamic & Quasi-static & Dynamic \\
\hline$L^{2}$-error( $\left.\overline{\text { Nusselt }}\right)$ & 52252.82 & 52391.22 & 52197.96 & 52249.35 \\
\hline $\bar{\eta}$ & $4.20 \times 10^{-3}$ & $1.56 \times 10^{-3}$ & $5.24 \times 10^{-3}$ & $4.26 \times 10^{-3}$ \\
\hline
\end{tabular}


Table 2: Differentially heated cavity AMR simulations driven by the subscales-based error and measured with the entropy measure.

\begin{tabular}{|l|c|c|c|c|}
\cline { 2 - 5 } \multicolumn{1}{c|}{} & \multicolumn{2}{c|}{ Residual } & \multicolumn{2}{c|}{ Orthogonal } \\
\cline { 2 - 5 } \multicolumn{1}{c|}{} & Quasi-static & Dynamic & Quasi-static & Dynamic \\
\hline$\overline{\text { Nusselt }}$ & 51432.90 & 51501.42 & 51425.60 & 50497.68 \\
\hline$L^{2}$-error( $\left.\overline{\text { Nuselt }}\right)$ & $1.98 \times 10^{-2}$ & $1.85 \times 10^{-2}$ & $1.99 \times 10^{-2}$ & $3.76 \times 10^{-2}$ \\
\hline $\bar{\eta}$ & $9.46 \times 10^{-4}$ & $9.31 \times 10^{-4}$ & $1.047 \times 10^{-3}$ & $9.58 \times 10^{-4}$ \\
\hline
\end{tabular}

\subsection{Supersonic flow over a flat plate}

In this fifth case, we test a viscid supersonic example: the $M=3$ flow over a flat plate problem. The problem domain is $[-0.1 L, L] \times[-H / 2, H / 2]$, with $L=1 \mathrm{~m}$, and $H=0.25 \mathrm{~m}$. The inlet flow conditions are fixed over the left-most boundary $\left(-0.1, x_{2}\right) \mathrm{m}$ as follows: a constant velocity prescription of $(3,0) \mathrm{m} / \mathrm{s}$, a constant density of $1 \mathrm{~kg} / \mathrm{m}^{3}$, and a constant temperature of $0.00248 \mathrm{~K}$. Zero flux conditions are imposed over the bottom boundary $\left(x_{1}<0,-H / 2\right) \mathrm{m}$ at the upstream. A no-slip condition for velocity, together with impermeable and adiabatic conditions, are specified for the plate surface $\left(x_{1}>0,-H / 2\right) \mathrm{m}$. Over the top boundary $\left(x_{1}, H / 2\right) \mathrm{m}$ the following conditions are prescribed: zero normal stress, a fixed value of $3 \mathrm{~m} / \mathrm{s}$ for the $x_{1}$-component of velocity, and a fixed density value of $1 \mathrm{~kg} / \mathrm{m}^{3}$. Lastly, free conditions are considered over the outflow wall, as the flow is supersonic. Viscosity and thermal conductivity are $\mu=3 \times 10^{-6}$ $\mathrm{kg} /(\mathrm{m} \mathrm{s})$ and $\lambda=4.23 \times 10^{-3} \mathrm{~W} /(\mathrm{m} \mathrm{K})$, respectively, defining a Reynolds number of Re $=10^{6}$

We run AMR simulations using the conservative variables formulation over a structured non-symmetric mesh composed of 11000 triangular $P_{1}$ elements, and achieve the mesh refinement with the subscales-based error until the transient convergence criterion is fulfilled. Figure 11 shows the density, momentum magnitude, and total energy results at the steady state. The flow is characterized by a thin boundary layer that separates from the plate, and by an oblique supersonic shock that is formed from the beginning of the plate to the outlet boundary. Results are presented for the refined mesh driven by the subscales-based error estimation measured with the scaled $L^{2}-$ norm. This flow corresponds accurately to the referenced result in [34], so that, the approximation achieved by the subscales-driven AMR of the compressible flow solver is satisfactory.

In this example, we prescribe the error tolerance to $\eta_{K}<10^{-8}$ for the scaled $L^{2}$-norm estimation, and to $\eta_{K}<10^{-10}$ for the entropy measure estimation. The refined meshes driven by subscales-based error measured with the scaled $L^{2}$-norm and with the entropy measure, are presented in Figures 12 and 13, respectively. The estimated error, which is below the prescribed tolerance for both simulations, is also presented on the right side of these figures. We observe that the refined meshes are able to represent the characteristic flow pattern of the viscous compressible supersonic flow; the boundary layer near the plate surface, and the supersonic shock are both correctly determined by the subscalesbased error estimator. Moreover, the mesh is especially refined near the flow singularity of the initial viscous point. This ability to reproduce the local phenomena related to the supersonic flow is expressed with both types of measures of the subscales-based error estimator. 


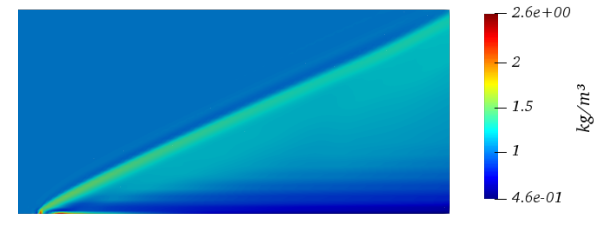

(a)

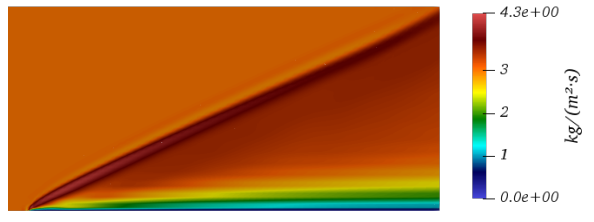

(b)

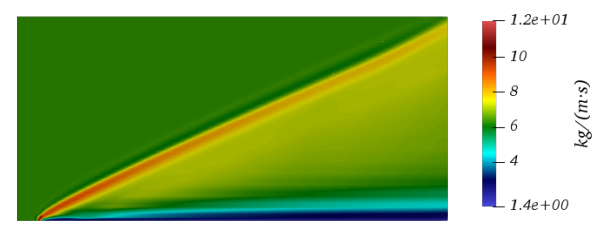

(c)

Figure 11: Flow over a flat plate results. Density, momentum magnitude, and total energy contours obtained using the refined mesh driven by the subscales-based error measured with the scaled $L^{2}-$ norm.
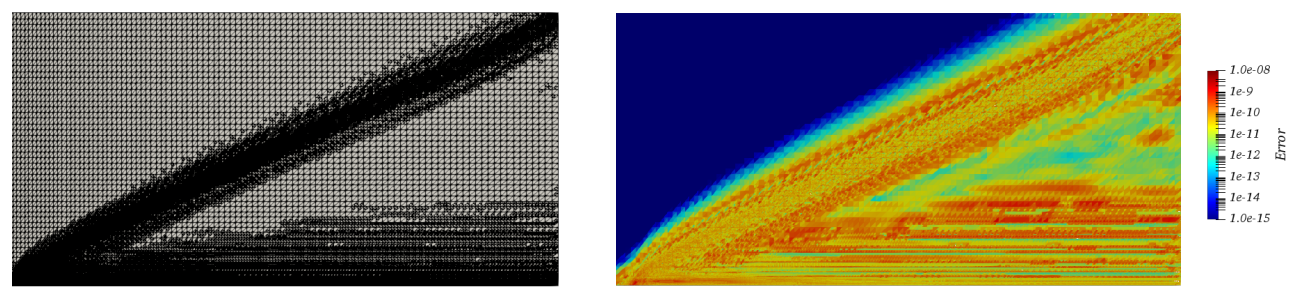

Figure 12: Flow over a flat plate results. Subscales-based error estimator measured with the scaled $L_{2}$-norm: refined mesh composed of 1558980 elements on the left, and estimated error over the refined mesh on the right.

\subsection{Supersonic flow past a cylinder}

The last numerical example that we solve is the supersonic flow past a cylinder problem at $\mathrm{Re}=2000$ and $\mathrm{M}=2$. The cylinder is defined to be infinitely long in the axial 

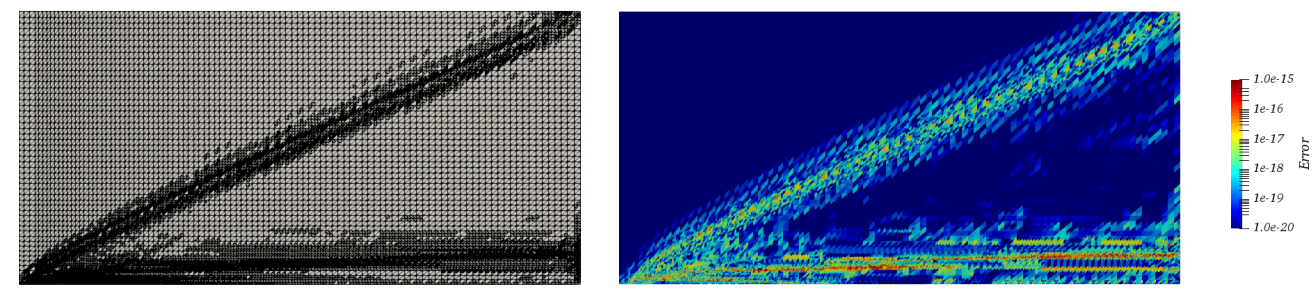

Figure 13: Flow over a flat plate results. Subscales-based error estimator measured with the entropy measure: refined mesh composed of 202806 elements on the left, and estimated error over the refined mesh on the right.

direction and immersed in a compressible viscous flow that impinges it uniformly. The domain for this problem is typically defined as a rectangular domain. Instead, we define a curve-shaped domain with the cylinder located in the center, in which the inlet and outlet curved boundaries intersect. Boundary conditions are set as follows. The flow is injected from the left wall with a uniform and constant velocity of $(1,0) \mathrm{m} / \mathrm{s}$, a temperature of $6.14 \times 10^{-4} \mathrm{~K}$, and a density of $1 \mathrm{~kg} / \mathrm{m}^{3}$. On the cylinder surface, a no-slip condition for velocity and an adiabatic condition for energy is imposed. Free conditions are considered over the outflow wall (as for supersonic flows). The physical properties are set to $\mu=0.0001 \mathrm{~kg} /(\mathrm{m} \mathrm{s})$ and $\lambda=0.14338 \mathrm{~kJ} /(\mathrm{m} \mathrm{K} \mathrm{s})$.

We depart from an initial unstructured mesh composed by $8141 P_{1}$ elements and run AMR simulations using the conservative variables formulation together with the subscales-based error estimation, measured both with the $L^{2}$-norm and the entropy measure, until the convergence criteria for advancing in time is satisfied. Figure 14 shows the steady state results for the supersonic flow past a cylinder. The solution in this figure is the one obtained with the refined mesh driven by the subscales-based error estimator and measured with the scaled $L^{2}-$ norm. We observe that the supersonic flow is composed by a strong shock at the upstream part of the cylinder, and by some oblique detached shock waves at the downstream part of the cylinder, as referenced in [33, 35]. The refinement gives an accurate resolution of the mesh at the thin shock layer of the supersonic expansion. It also gives correct results where gradients of the solution are not too sharp, such as for the weak tail shock that is formed in the wake structure.

Figures 15 and 16 display the comparison between the original unstructured mesh and the refined meshes driven by the error estimators measured with the scaled $L^{2}-$ norm and with the entropy measure, respectively. In both AMR simulations the tolerance is fixed depending on the selected norm; in the case of the scaled $L^{2}$-norm we set the tolerance to $\eta_{K}<10^{-5}$, and in the case of the entropy measure we fix it to $\eta_{K}<10^{-8}$. The estimated error distribution, which is below the tolerance in both simulations, is also presented on the right side of those figures.

We observe that resolution of flow singularities and shocks, including the upstream supersonic shock, the description of boundary layers near the cylinder surface, and the wake structure of the flow, are described correctly by the refined mesh. In the case of 
the $L^{2}$-norm results, the subscales-based error estimation is taking effect mostly at the supersonic shock, and at the boundary layer near the cylinder surface. It can also be seen that the upstream supersonic shock structures (but also downstream) are greatly characterized by the use of this norm. It is worth to comment that the reference velocity of the scaled $L^{2}$-norm (42) designed to be of the order of $u_{0} \approx|\boldsymbol{u}|+c$ gives accurate definitions of the error estimation. In the case of the subscales-based error estimator measured with the entropy measure, the refinement is acting homogeneously through the downstream part of the flow, so that, the resulting mesh is strongly refined at the wake structure behind the cylinder (even as much as for the supersonic shock).
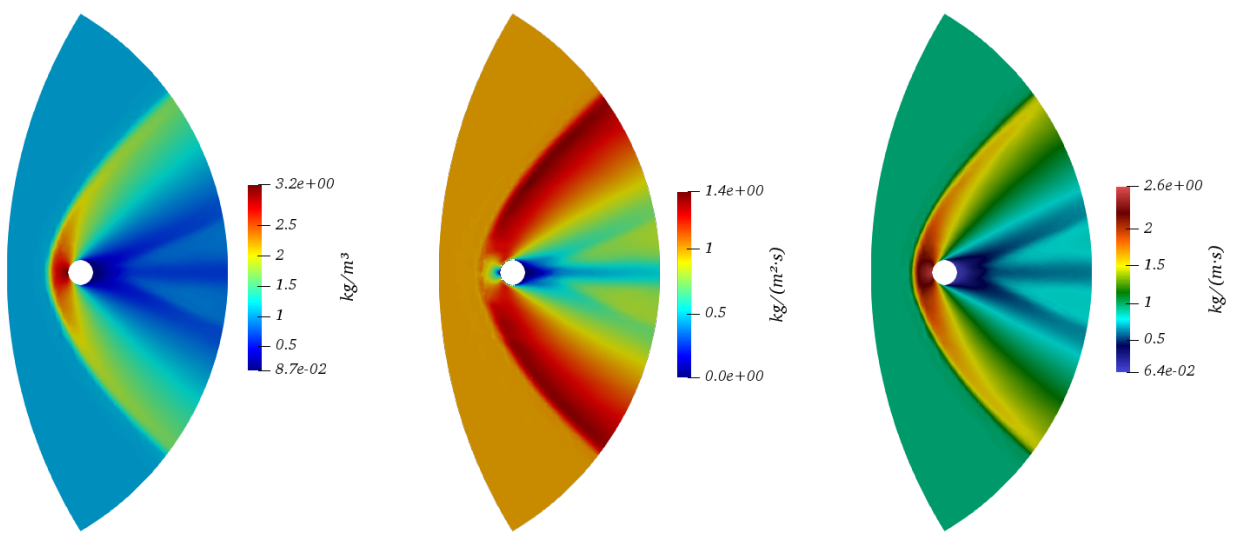

Figure 14: Supersonic flow past a cylinder results. Density, momentum magnitude, and total energy steady solution using the refined mesh driven by the subscales-based error estimator measured with the scaled $L_{2}$-norm.

\section{Conclusions}

In this work, we have used the variational subscales as an error estimator for the adaptive mesh refinement of compressible flow simulations. The estimator includes both the subscales at the element boundaries and in the interior of the elements. These subscales are defined as orthogonal, dynamic and non-linear. Appropriate measures, namely, a scaled $L^{2}$-norm, and an entropy measure, have been used for composing the contribution of the subscales into a single error estimate.

The method has been tested in subsonic and supersonic compressible flow examples, both with the conservative variables formulation and with the primitive variables formulation. In all numerical examples, the local error has been measured using the subscales-based estimator, and the AMR has been performed leading to an equally distributed estimated error (below some prescribed tolerance). The error estimation given by the subscales has demonstrated to provide accurate information about the discretization error in an explicit fashion, this is, without having to estimate an overall error in the solution. This methodology has proven to give an efficient refined mesh with regard to the estimated error and the computational effort. 

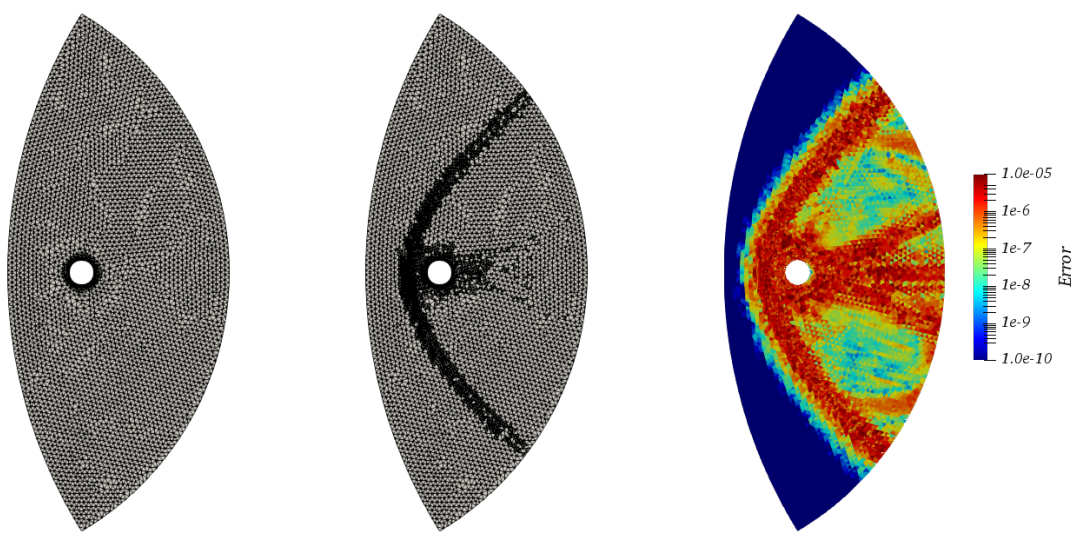

Figure 15: Supersonic flow past a cylinder results. Subscales-based error estimator measured with the scaled $L_{2}$-norm: initial unstructured mesh composed by $8141 P_{1}$ elements on the left, refined mesh composed of $15613 P_{1}$ elements on the center, and estimated error over the refined mesh on the right.
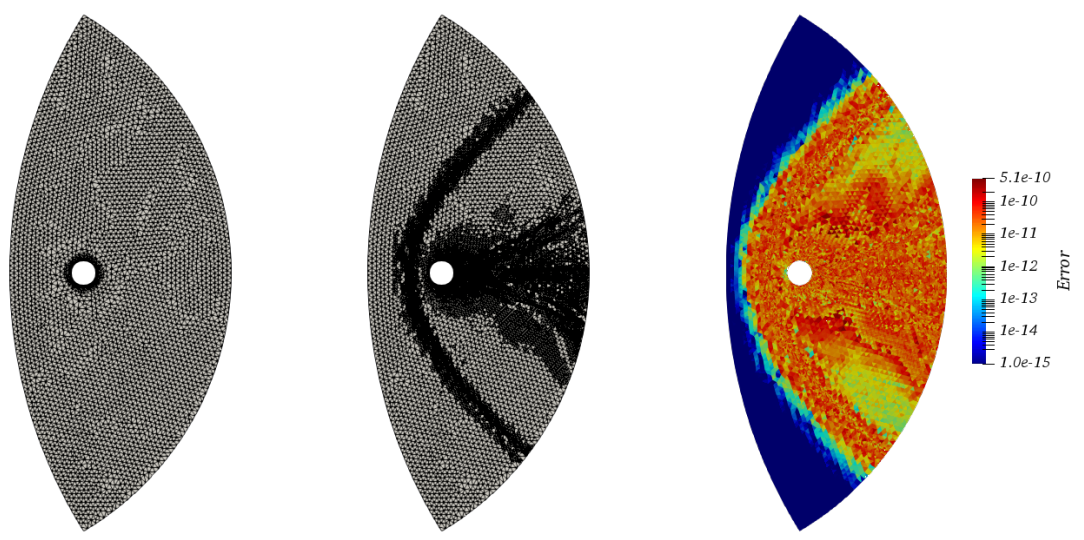

Figure 16: Supersonic flow past a cylinder results. Subscales-based error estimator measured with the entropy measure: initial unstructured mesh composed by $8141 P_{1}$ elements on the left, refined mesh composed of $61020 P_{1}$ elements on the center, and estimated error over the refined mesh on the right.

\section{Acknowledgements}

C. Bayona acknowledges the doctoral scholarship received from the Colombian GovernmentColciencias. R. Codina acknowledges the support received from the ICREA Acadèmia Research Program of the Catalan Government. J. Baiges acknowledges the support of the Spanish Government through the Ramón y Cajal grant RYC-2015-17367. This work is partially funded through the ELASTIC-FLOW project, Ref. DPI2015-67857-R of the Spanish Government. The support of the European PRACE computational network and the RES-Barcelona Supercomputing center in the development of the distributed 
memory adaptive mesh refinement algorithms is acknowledged.

\section{References}

[1] T. Hughes, "Multiscale phenomena: Green's functions, the Dirichlet-to-Neumann formulation, subgrid scale models, bubbles and the origins of stabilized methods," Comput. Methods. Appl. Mech. \& Eng., vol. 127, no. 1, pp. 387-401, 1995.

[2] R. Codina, S. Badia, J. Baiges, and J. Principe, "Variational multiscale methods in computational fluid dynamics," in Encyclopedia of computational mechanics, E. Stein, R. de Borst, and T. J. Hughes, Eds., Wiley Online Library.

[3] R. Codina, "Stabilized finite element approximation of transient incompressible flows using orthogonal subscales," Comput. Methods. Appl. Mech. 6 Eng., vol. 191, no. 39, pp. 4295-4321, 2002 .

[4] T. J. R. Hughes, V. M. Calo, and G. Scovazzi, "Variational and multiscale methods in turbulence," in Mechanics of the 21st Century, Springer, 2005, pp. 153-163.

[5] C. Bayona, J. Baiges, and R. Codina, "Variational multi-scale approximation of the one-dimensional forced Burgers equation: The role of orthogonal sub-grid scales in turbulence modeling," International Journal for Numerical Methods in Fluids, n/a-n/a, ISSN: 1097-0363. DOI: 10.1002/fld.4420. [Online]. Available: http://dx.doi.org/10.1002/fld.4420.

[6] D. Irisarri and G. Hauke, "A posteriori pointwise error computation for 2-D transport equations based on the variational multiscale method," Comput. Methods. Appl. Mech. \&f Eng., vol. 311, pp. 648-670, 2016.

[7] M. G. Larson and A. Målqvist, "Adaptive variational multiscale methods based on a posteriori error estimation: Energy norm estimates for elliptic problems," Comput. Methods. Appl. Mech. EG Eng., vol. 196, no. 21, pp. 2313-2324, 2007.

[8] A. ElSheikh, S. Chidiac, and W. Smith, "A posteriori error estimation based on numerical realization of the variational multiscale method," Comput. Methods. Appl. Mech. EG Eng., vol. 197, no. 45, pp. 3637-3656, 2008.

[9] P. Ladeveze and D. Leguillon, "Error estimate procedure in the finite element method and applications," SIAM Journal on Numerical Analysis, vol. 20, no. 3, pp. 485-509, 1983.

[10] S. Berrone, "Robustness in a posteriori error analysis for FEM flow models," Numerische Mathematik, vol. 91, no. 3, pp. 389-422, 2002.

[11] G. Hauke, M. H. Doweidar, and S. Fuentes, "Mesh adaptivity for the transport equation led by variational multiscale error estimators," International Journal for Numerical Methods in Fluids, vol. 69, no. 12 , pp. 1835-1850, 2012.

[12] G. Hauke, D. Fuster, and F. Lizarraga, "Variational multiscale a posteriori error estimation for systems: The Euler and Navier-Stokes equations," Comput. Methods. Appl. Mech. ES Eng., vol. 283, pp. 1493-1524, 2015.

[13] R. Rossi, J Cotela, N. M. Lafontaine, P. Dadvand, and S. R. Idelsohn, "Parallel adaptive mesh refinement for incompressible flow problems," Computers E Fluids, vol. 80, pp. 342-355, 2013.

[14] R. Hartmann, "Adaptive discontinuous Galerkin methods with shock-capturing for the compressible Navier-Stokes equations," International Journal for Numerical Methods in Fluids, vol. 51, no. 9-10, pp. 1131-1156, 2006.

[15] K. J. Fidkowski and D. L. Darmofal, "A triangular cut-cell adaptive method for high-order discretizations of the compressible Navier-Stokes equations," Journal of Computational Physics, vol. 225, no. 2, pp. 1653-1672, 2007.

[16] M. Woopen, A. Balan, G. May, and J. Schütz, "A comparison of hybridized and standard DG methods for target-based hp-adaptive simulation of compressible flow," Computers EG Fluids, vol. 98, pp. 3-16, 2014.

[17] M. Kouhi, E. Oñate, and D. Mavriplis, "Adjoint-based adaptive finite element method for the compressible Euler equations using finite calculus," Aerospace Science and Technology, vol. 46, pp. $422-435,2015$. 
[18] B. R. Ahrabi, W. K. Anderson, and J. C. Newman, "An adjoint-based hp-adaptive stabilized finite-element method with shock capturing for turbulent flows," Comput. Methods. Appl. Mech. E Eng., vol. 318, pp. 1030-1065, 2017.

[19] J. Oden, L Demkowicz, W Rachowicz, and T. Westermann, "A posteriori error analysis in finite elements: The element residual method for symmetrizable problems with applications to compressible Euler and Navier-Stokes equations," Comput. Methods. Appl. Mech. ES Eng., vol. 82, no. 1-3, pp. 183-203, 1990.

[20] W Rachowicz, "An anisotropic h-adaptive finite element method for compressible Navier-Stokes equations," Comput. Methods. Appl. Mech. \& Eng., vol. 146, no. 3-4, pp. 231-252, 1997.

[21] G. Hauke, M. H. Doweidar, and M. Miana, "The multiscale approach to error estimation and adaptivity," Comput. Methods. Appl. Mech. E Eng., vol. 195, no. 13, pp. 1573-1593, 2006.

[22] R. Codina, "Stabilized finite element approximation of transient incompressible flows using orthogonal subscales," Comput. Methods. Appl. Mech. Es Eng., vol. 191, no. 39, pp. 4295-4321, 2002.

[23] J. Baiges and R. Codina, "Variational Multiscale error estimators for solid mechanics adaptive simulations: An Orthogonal Subgrid Scale approach," Comput. Methods. Appl. Mech. E Eng., vol. 325, pp. 37-55, 2017.

[24] R. Codina, J. Principe, O. Guasch, and S. Badia, "Time dependent subscales in the stabilized finite element approximation of incompressible flow problems," Comput. Methods. Appl. Mech. EG Eng., vol. 196, no. 21, pp. 2413-2430, 2007.

[25] R. Codina, "Finite element approximation of the three-field formulation of the Stokes problem using arbitrary interpolations," SIAM Journal on Numerical Analysis, vol. 47, no. 1, pp. 699-718, 2009.

[26] C. Bayona, J. Baiges, and R. Codina, "Variational multi-scale finite element approximation of the compressible Navier-Stokes equations," International Journal of Numerical Methods for Heat 6 Fluid Flow, vol. 26, no. 3/4, pp. 1240-1271, 2016.

[27] R. Codina, J. Principe, and J. Baiges, "Subscales on the element boundaries in the variational two-scale finite element method," Comput. Methods. Appl. Mech. EG Eng., vol. 198, no. 5, pp. 838$852,2009$.

[28] J. Baiges and C. Bayona, "Refficientlib: An Efficient Load-Rebalanced Adaptive Mesh Refinement Algorithm for High-Performance Computational Physics Meshes," SIAM Journal on Scientific Computing, vol. 39, no. 2, pp. C65-C95, 2017.

[29] C. Bayona, J. Baiges, and R. Codina, "Solution of low Mach number aeroacoustic flows using a Variational Multi-scale finite element formulation of the compressible Navier-Stokes equations written in primitive variables," Comput. Methods. Appl. Mech. EG Eng., Submitted.

[30] L. El Alaoui and A. Ern, "Residual and hierarchical a posteriori error estimates for nonconforming mixed finite element methods," ESAIM: Mathematical Modelling and Numerical Analysis, vol. 38, no. 6, 903-929, 2004.

[31] O. C. Zienkiewicz and J. Z. Zhu, "A simple error estimator and adaptive procedure for practical engineerng analysis," International Journal for Numerical Methods in Engineering, vol. 24, no. 2, pp. 337-357, 1987.

[32] R. Codina, "An iterative penalty method for the finite element solution of the stationary NavierStokes equations," Comput. Methods. Appl. Mech. \& Eng., vol. 110, no. 3-4, pp. 237-262, 1993.

[33] S. Mittal and T Tezduyar, "A unified finite element formulation for compressible and incompressible flows using augmented conservation variables," Comput. Methods. Appl. Mech. EG Eng., vol. 161, no. 3-4, pp. 229-243, 1998.

[34] V. Kotteda and S. Mittal, "Stabilized finite element computation of compressible flow with linear and quadratic interpolation functions," International Journal for Numerical Methods in Fluids, vol. 75, no. 4, pp. 273-294, 2014.

[35] F. Rispoli and R. Saavedra, "A stabilized finite element method based on sgs models for compressible flows," Comput. Methods. Appl. Mech. EG Eng., vol. 196, no. 1, pp. 652-664, 2006. 


\section{Appendix A: Jacobian matrices}

The formulation using conservative variables $\boldsymbol{U}=\left(\rho, \boldsymbol{m}, e_{\mathrm{tot}}\right)^{\top}$ includes a transient Jacobian matrix $\boldsymbol{A}_{0}$ equal to the $(d+2) \times(d+2)$ identity matrix, and a convective Jacobian matrix $\boldsymbol{A}_{j}(\boldsymbol{U})$ of the form:

$$
\boldsymbol{A}_{j}(\boldsymbol{U})=\left[\begin{array}{ccc}
0 & \boldsymbol{e}_{j}^{\top} & 0 \\
\boldsymbol{u} u_{j}+a_{1} \boldsymbol{e}_{j} & \boldsymbol{I} u_{j}+\boldsymbol{u} \otimes \boldsymbol{e}_{j}-(\gamma-1)\left(\boldsymbol{e}_{j} \otimes \boldsymbol{e}_{j}\right) \boldsymbol{u}^{\top} & (\gamma-1)\left(\boldsymbol{e}_{j} \otimes \boldsymbol{e}_{j}\right) \\
\left(a_{1}-a_{2}\right) u_{j} & -(\gamma-1) \boldsymbol{u}^{\top} u_{j}+a_{2} \boldsymbol{e}_{j}^{\top} & \gamma u_{j}
\end{array}\right],
$$

for $j=1, . ., d$, Let us denote by $\mathbf{0}$ the vector of $\mathbb{R}^{d}$ with zero in all its components, and $\boldsymbol{e}_{i}$ the unit vector in the $i$-th direction. In the previous definition the thermodynamic relations $a_{1}$ and $a_{2}$ stand for $a_{1}=\frac{1}{2}(\gamma-1)|\boldsymbol{u}|^{2}$, and $a_{2}=\frac{1}{\rho}\left(e_{\text {tot }}+p\right)$, respectively. Using the viscous stress tensor and heat flux vector definitions based on conservative variables and the ideal gas law, each matrix component in the Jacobian diffusive matrix $\mathbf{K}_{k j}(\mathbf{U})$ is constructed as

$$
\begin{aligned}
& \boldsymbol{K}_{j j}(\boldsymbol{U})=\left[\begin{array}{ccc}
0 & \mathbf{0}^{\top} & 0 \\
-\nu \boldsymbol{u} & \nu \boldsymbol{I} & \mathbf{0} \\
(\alpha-\nu)|\boldsymbol{u}|^{2}-\frac{1}{\rho} \alpha e_{\text {tot }} & (\nu-\alpha) \boldsymbol{u}^{\top} & \alpha
\end{array}\right] \quad \text { for } j=1, . ., d \text {, and } \\
& \boldsymbol{K}_{k j}(\boldsymbol{U})=\left[\begin{array}{ccc}
0 & \mathbf{0}^{\top} & 0 \\
-\nu u_{k} \boldsymbol{e}_{j}+\frac{2}{3} \nu u_{j} \boldsymbol{e}_{k} & \nu \boldsymbol{e}_{j} \otimes \boldsymbol{e}_{k}-\frac{2}{3} \nu \boldsymbol{e}_{k} \otimes \boldsymbol{e}_{j} & \mathbf{0} \\
-\frac{1}{3} \nu u_{k} u_{j} & \nu u_{j} \boldsymbol{e}_{k}^{\top}-\frac{2}{3} \nu u_{k} \boldsymbol{e}_{j}^{\top} & 0
\end{array}\right]
\end{aligned}
$$

for $k, j=1, . ., d$, with $k \neq j$.

In the case of the compressible Navier-Stokes equations written in primitive variables $\boldsymbol{U}=(p, \boldsymbol{u}, T)^{\top}, \boldsymbol{A}_{0}(\boldsymbol{U})$ and the Jacobian matrices $\boldsymbol{A}_{j}(\boldsymbol{U}), j=1, \ldots, d$, can be formulated as functions of thermodynamic coefficients by supposing that the fluid is divariant and an ideal gas:

$$
\begin{aligned}
\boldsymbol{A}_{0}(\boldsymbol{U}) & =\left[\begin{array}{ccc}
\rho \beta_{t} & \mathbf{0}^{\top} & -\rho \alpha_{p} \\
\rho \beta_{t} \boldsymbol{u} & \rho \boldsymbol{I} & -\rho \alpha_{p} \boldsymbol{u} \\
\rho \beta_{t} a_{1}-\alpha_{p} T & \rho \boldsymbol{u}^{\top} & -\rho \alpha_{p} a_{1}+\rho c_{p}
\end{array}\right], \\
\boldsymbol{A}_{j}(\boldsymbol{U}) & =\left[\begin{array}{ccc}
\rho \beta_{t} u_{j} & \rho \boldsymbol{e}_{j}^{\top} & -\rho \alpha_{p} u_{j} \\
\rho \beta_{t} \boldsymbol{u} u_{j}+\boldsymbol{e}_{j} & \rho \mathbf{I} u_{j}+\rho \boldsymbol{u} \otimes \boldsymbol{e}_{j} & -\rho \alpha_{p} \boldsymbol{u} u_{j} \\
\left(\rho \beta_{t} a_{1}-\alpha_{p} T+1\right) u_{j} & \rho \boldsymbol{u}^{\top} u_{j}+\rho a_{1} \boldsymbol{e}_{j}^{\top} & \left(-\rho \alpha_{p} a_{1}+\rho c_{p}\right) u_{j}
\end{array}\right],
\end{aligned}
$$

for $j=1, . ., d$. . The thermodynamic relation $a_{1}$ stands for $a_{1}=c_{v} T+p / \rho+|\boldsymbol{u}|^{2} / 2$, $\alpha_{p}$ is the volume expansivity, and $\beta_{t}$ is the isothermal compressibility. Additionally, the diffusive matrices $\boldsymbol{K}_{k j}(\boldsymbol{U})$ are constructed as

$$
\begin{aligned}
\boldsymbol{K}_{j j}(\boldsymbol{U}) & =\left[\begin{array}{ccc}
0 & \mathbf{0}^{\top} & 0 \\
\mathbf{0} & \mu \boldsymbol{I}+\frac{1}{3} \mu \boldsymbol{e}_{j} \otimes \boldsymbol{e}_{j} & \mathbf{0} \\
0 & \mu \boldsymbol{u}^{\top}+\frac{1}{3} \mu u_{j} \boldsymbol{e}_{j}^{\top} & \lambda
\end{array}\right] \quad \text { for } j=1, . ., d, \text { and } \\
\boldsymbol{K}_{k j}(\boldsymbol{U}) & =\left[\begin{array}{ccc}
0 & \mathbf{0}^{\top} & 0 \\
\mathbf{0} & \mu \boldsymbol{e}_{j} \otimes \boldsymbol{e}_{k}-\frac{2}{3} \mu \boldsymbol{e}_{k} \otimes \boldsymbol{e}_{j} & \mathbf{0} \\
0 & \mu u_{j} \boldsymbol{e}_{k}^{\top}-\frac{2}{3} \mu u_{k} \boldsymbol{e}_{j}^{\top} & 0
\end{array}\right] \quad \text { for } k, j=1, . ., d, \text { with } k \neq j .
\end{aligned}
$$

